\title{
Dynamical evolution of a magnetic cloud from the Sun to $5.4 \mathrm{AU}$
}

\author{
M. S. Nakwacki ${ }^{1,2}$, S. Dasso ${ }^{2,3}$, P. Démoulin ${ }^{4}$, C. H. Mandrini2,5 ${ }^{2,5}$ and A. M. Gulisano ${ }^{2,5}$ \\ ${ }^{1}$ Instituto de Astronomia, Geofísica e Ciências Atmosféricas, Universidade de São Paulo, Brazil \\ e-mail: sole@astro.iag.usp.br \\ 2 Instituto de Astronomía y Física del Espacio, CONICET-UBA, Argentina \\ 3 Departamento de Física, Facultad de Ciencias Exactas y Naturales, UBA, Argentina \\ ${ }^{4}$ Observatoire de Paris, LESIA, UMR 8109 CNRS, 92195 Meudon Principal Cedex, France \\ 5 Facultad de Ciencias Exactas y Naturales, FCEN, UBA, Argentina
}

Received 1 October 2010 / Accepted 28 July 2011

\begin{abstract}
Context. Significant quantities of magnetized plasma are transported from the Sun to the interstellar medium via interplanetary coronal mass ejections (ICMEs). Magnetic clouds (MCs) are a particular subset of ICMEs, forming large-scale magnetic flux ropes. Their evolution in the solar wind is complex and mainly determined by their own magnetic forces and the interaction with the surrounding solar wind.

Aims. Magnetic clouds are strongly affected by the surrounding environment as they evolve in the solar wind. We study expansion of MCs, its consequent decrease in magnetic field intensity and mass density, and the possible evolution of the so-called global idealMHD invariants.

Methods. In this work we analyze the evolution of a particular MC (observed in March 1998) using in situ observations made by two spacecraft approximately aligned with the Sun, the first one at 1 AU from the Sun and the second one at 5.4 AU. We describe the magnetic configuration of the MC using different models and compute relevant global quantities (magnetic fluxes, helicity, and energy) at both heliodistances. We also tracked this structure back to the Sun, to find out its solar source.

Results. We find that the flux rope is significantly distorted at 5.4 AU. From the observed decay of magnetic field and mass density, we quantify how anisotropic is the expansion and the consequent deformation of the flux rope in favor of a cross section with an aspect ratio at $5.4 \mathrm{AU}$ of $\approx 1.6$ (larger in the direction perpendicular to the radial direction from the Sun). We quantify the ideal-MHD invariants and magnetic energy at both locations, and find that invariants are almost conserved, while the magnetic energy decays as expected with the expansion rate found.

Conclusions. The use of MHD invariants to link structures at the Sun and the interplanetary medium is supported by the results of this multi-spacecraft study. We also conclude that the local dimensionless expansion rate, which is computed from the velocity profile observed by a single-spacecraft, is very accurate for predicting the evolution of flux ropes in the solar wind.
\end{abstract}

Key words. magnetohydrodynamics (MHD) - magnetic fields - solar wind - Sun: heliosphere - Sun: magnetic topology Sun: coronal mass ejections (CMEs)

\section{Introduction}

Coronal mass ejections (CMEs) are explosive events that release energy in the solar atmosphere. The interplanetary counterparts of CMEs are solar wind (SW) structures known as interplanetary coronal mass ejections (ICMEs). Among them there is a subset, called magnetic clouds (MCs), which exhibit a smooth rotation of the magnetic field direction through a large angle, enhanced magnetic field strength, low proton temperature and a low proton plasma beta, $\beta_{\mathrm{p}}$. MCs are formed by large-scale magnetic flux ropes carrying a large amount of magnetic helicity, magnetic flux, and energy away from the Sun. The main characteristics of these structures have been enumerated by Burlaga \& Klein (1980).

Several authors have consider MCs as static flux ropes (see, e.g., Goldstein 1983; Burlaga 1988; Lepping et al. 1990; Burlaga 1995; Lynch et al. 2003). Their magnetic fields have frequently been modeled using the Lundquist's model (Lundquist 1950), which considers a static and axially symmetric linear force-free magnetic configuration. Many deviations from this model have also been studied: e.g., nonlinear force-free fields (Farrugia et al. 1999), nonforce-free fields (Mulligan et al. 1999b; Hidalgo et al. 2002; Cid et al. 2002), and several noncylindrical models (Hu \& Sonnerup 2001; Vandas \& Romashets 2002; Démoulin \& Dasso 2009b), all of them being static. These models are recurrently used to fit in situ magnetic field measurements within MCs to reconstruct the whole flux rope structure. These techniques have also been tested by replacing the observations by the local values found in a numerical simulation, and the output of the models has been compared to the known original full simulation (Riley et al. 2004). The results of these comparisons show that these in situ techniques can reproduce the magnetic structures relatively well when the spacecraft is crossing the $\mathrm{MC}$ near its main axis.

In many cases, MCs present clear characteristics of expansion (e.g., Lepping et al. 2003, 2008), so several dynamical models have been developed to describe these clouds during their observation time. Some of these flux rope models suppose a circular cross-section with only a radial expansion (Farrugia et al. 1993; Osherovich et al. 1993b; Farrugia et al. 1997; Shimazu \& Marubashi 2000; Nakwacki et al. 2008b), while other models 
include expansion in both directions, radial and axial (Shimazu \& Vandas 2002; Berdichevsky et al. 2003; Démoulin \& Dasso 2009a; Nakwacki et al. 2008a). A dynamical model with an elliptical shape has been derived by Hidalgo (2003), while a model of the expansion with an anisotropic self-similar expansion in three orthogonal directions was worked out by Démoulin et al. (2008).

From single-spacecraft observations we cannot directly infer the global structure of the flux ropes and their evolution through the interplanetary medium because they are one single point local measurements. Several strategies are used to derive more information on MCs with multi-spacecraft data, as follows.

With two spacecraft located at a similar distance from the Sun and separated by a distance close to that of the cross-section of the encountered flux rope, in situ observations provide data at different parts of the flux rope, which are barely affected by its evolution. This is used to test the technique by computing the magnetic field in the cross section from the data of one spacecraft and/or to have a more accurate reconstruction of the magnetic field (Mulligan \& Russell 2001; Liu et al. 2008; Kilpua et al. 2009; Möstl et al. 2009).

When the two spacecraft positions are viewed from the Sun with a significant angle, typically in the interval $\left[10^{\circ}, 80^{\circ}\right]$, one can usually derive an estimation of the extension of the flux rope or, at least, an estimation of the extension of the perturbation (e.g., the front shock) induced by the propagation of the flux rope in the interplanetary medium (Cane et al. 1997; Mulligan \& Russell 2001; Reisenfeld et al. 2003). A larger number of spacecraft permits constraining the evolving magnetic structure more, such as in the case analyzed by Burlaga et al. (1981). Such studies have been extended to cases where the spacecraft are separated well in solar distance with one spacecraft near Earth and the other one at a few AUs (Hammond et al. 1995; Gosling et al. 1995; Liu et al. 2006; Foullon et al. 2007; Rodriguez et al. 2008). They show that large MCs/ICMEs have large-scale effects on the heliosphere (e.g., both at low and high latitudes).

When the two spacecraft are separated by spatial scales on the order of one or several AUs, the above analysis should take the evolution of the MC with solar distance into account. This also implies a more difficult association of the in situ observations on both spacecraft. Numerical simulations are then useful tools for checking whether the events observed on each spacecraft are in fact a unique event (e.g., Riley et al. 2003). Since MCs are moving mostly radially away from the Sun, the radial alignment (line-up) of two spacecraft is a major opportunity to study the radial evolution of an MC, as the MC is crossed at a similar location by the two spacecraft. However, it is not common to find events observed by two nearly radially aligned spacecraft. One case was observed by Helios- 1,2 close to $1 \mathrm{AU}$ and later by Voyager-1, 2 at $2 \mathrm{AU}$, with an angular separation from the Sun of about $10^{\circ}$ (resp. $23^{\circ}$ ) between Helios1 (resp. Helios-2) and both Voyager-1,2 (Burlaga et al. 1981; Osherovich et al. 1993a). A second case was observed by Wind and NEAR spacecraft with an angular separation from the Sun of about $1^{\circ}$ and a ratio of solar distances of 1.2 (Mulligan et al. 1999a). A third case was observed by ACE and NEAR spacecraft with an angular separation from the Sun of about $2^{\circ}$ and a ratio of solar distances of 1.8 (Mulligan et al. 2001). Finally, a fourth case was observed by ACE and Ulysses spacecraft with an angular separation from the Sun of about $6^{\circ}$ and a ratio of solar distances of 5.4 (Skoug et al. 2000; Du et al. 2007). This last case has the advantage of a larger radial separation, so that the evolution has a stronger effect. This is the MC selected for a deeper study in this paper.
From the in situ data at different solar distances of the same $\mathrm{MC}$, one can directly infer the evolution of the magnetic field and plasma quantities. Such radial evolution is otherwise available only from a statistic analysis of a large number of MCs observed individually at various distances (Liu et al. 2005; Wang et al. 2005a; Leitner et al. 2007; Gulisano et al. 2010), with possible bias coming from the selection of MCs with different properties. Another application of line-up spacecraft is to derive the evolution of global magnetohydrodynamic (MHD) quantities, such as magnetic flux and magnetic helicity (Dasso 2009). They are the main quantities for testing whether the flux rope simply expands or if a significant part reconnects with the SW field. These global quantities permit also a quantitative link to the related solar event (Mandrini et al. 2005; Luoni et al. 2005; Rodriguez et al. 2008), and set constraints on the physical mechanism of the associated CME launch (Webb et al. 2000; Attrill et al. 2006; Qiu et al. 2007).

In this paper we further analyze the evolution of an $\mathrm{MC}$ observed at two different heliodistances, 1 and 5.4 AU (Skoug et al. 2000; Du et al. 2007). This MC was selected because this is to our knowledge the best line-up observations of an MC between ACE and Ulysses spacecraft. The observations are summarized in Sect. 2. The velocity and magnetic models used to complement the observations are described in Sect. 3. This spacecraft line-up is an opportunity to follow the evolution of the flux rope and, in particular, the global MHD quantities such as magnetic helicity and flux (Sect. 4). We relate this MC to its solar source in Sect. 5. This complements our understanding of the magnetic field evolution. Finally, we discuss our results and conclude in Sect. 6.

\section{Observations}

\subsection{Instruments and spacecraft}

We analyzed data sets for SW plasma and magnetic field from ACE and Ulysses spacecraft. We used the Magnetic Field Experiment (MAG, Smith et al. 1998) with a temporal cadence of 16 seconds and the Solar Wind Electron Proton Alpha Monitor (SWEPAM, McComas et al. 1998) with a temporal cadence of 64 seconds for ACE spacecraft. For Ulysses spacecraft, we used Vector Helium Magnetometer (VHM, Balogh et al. 1992) for magnetic field observations with a temporal cadence of 1 second and solar Wind observations over the Poles of the Sun (SWOOPS, Bame et al. 1992) for plasma observations with a temporal cadence of 4 min.

When the MC passed through Earth (March 5, 1998) ACE was located at $\approx 1 \mathrm{AU}$ in the ecliptic plane and in a longitude of $164^{\circ}$ in the solar ecliptic (SE) coordinate system. When the cloud was observed by Ulysses (March 25, 1998), this spacecraft was located at 5.4 AU from the Sun and very near the ecliptic plane; in particular, it was at a latitude of $2^{\circ}$ and at a longitude of $158^{\circ}$ in the SE system. Thus, the position of both spacecraft differs in $2^{\circ}$ for latitude and in $6^{\circ}$ for longitude (Skoug et al. 2000; $\mathrm{Du}$ et al. 2007). This angular separation corresponds to a separation distance (perpendicular to the radial direction to the Sun) of $\approx 0.6 \mathrm{AU}$ at the location of Ulysses. This very good alignment between the Sun and both points of observation of the same object gives us a unique opportunity to observe the same $\mathrm{MC}$ at two different evolution stages in the heliosphere.

\subsection{Coordinate systems}

We analyze ACE data in geocentric solar ecliptic (GSE) system of reference $\left(\hat{\boldsymbol{x}}_{\mathrm{GSE}}, \hat{\boldsymbol{y}}_{\mathrm{GSE}}, \hat{\boldsymbol{z}}_{\mathrm{GSE}}\right)$, where $\hat{\boldsymbol{x}}_{\mathrm{GSE}}$ points from the 

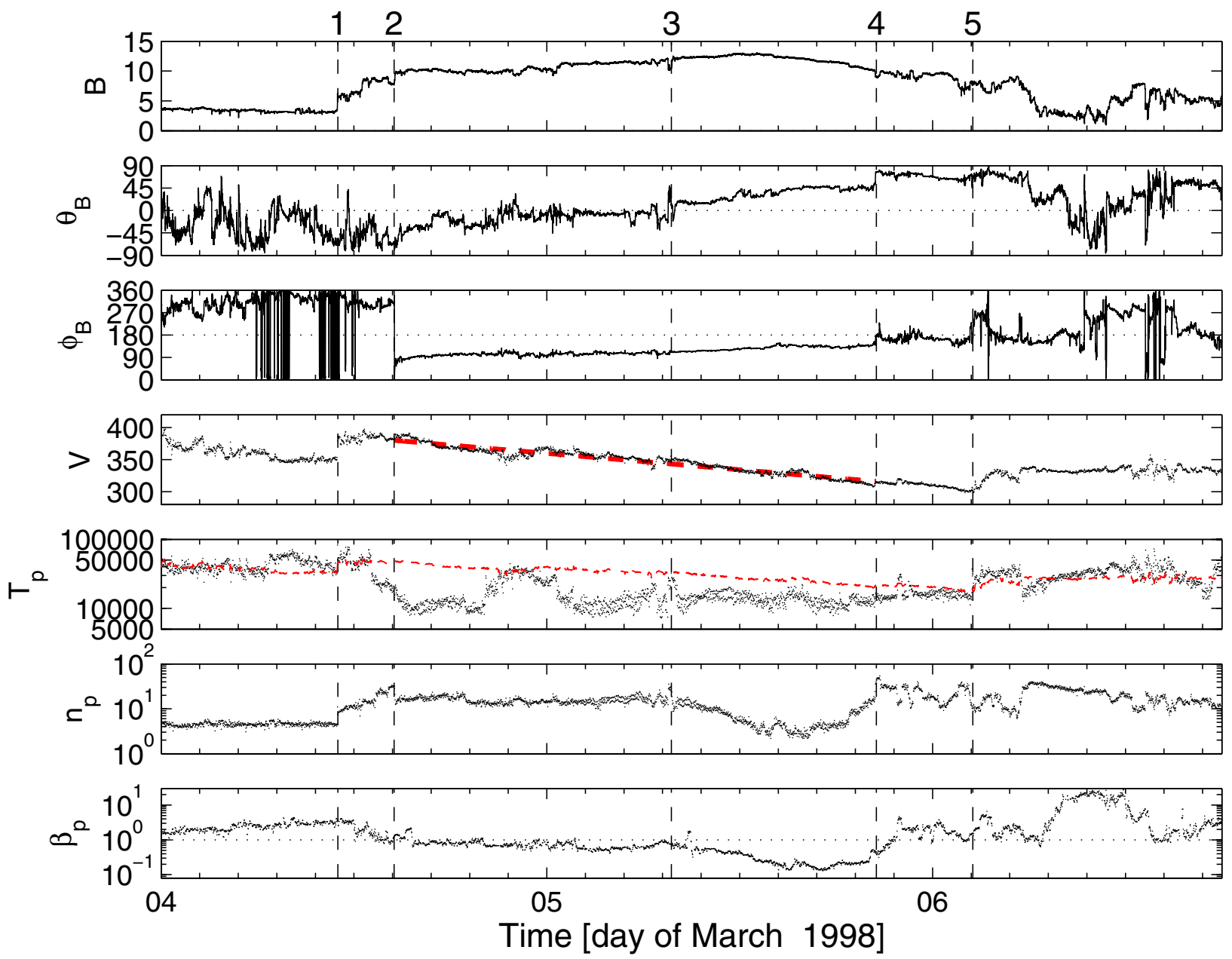

Fig. 1. In situ plasma and magnetic field of the ICME observed in March 1998 by ACE located at $\approx 1$ AU from the Sun. From top to bottom: absolute value of the magnetic field $(B=|\boldsymbol{B}|$, in $\mathrm{nT})$, magnetic field vector orientation (GSE): latitude $\left(\theta_{B}\right)$ and longitude $\left(\phi_{B}\right)$, bulk velocity $(V$, in $\mathrm{km} \mathrm{s}^{-1}$ ) including in black the fitted straight line for the MC range (see Sect. 4.5), the expected (continuous black line) and observed (dots) proton temperature $\left(T_{\mathrm{p}}\right.$, in $\left.\mathrm{K}\right)$, proton density $\left(n_{\mathrm{p}}\right.$, in $\left.\mathrm{cm}^{-3}\right)$, and proton plasma beta $\left(\beta_{\mathrm{p}}\right)$. Vertical lines mark different interfaces separating different plasma regions (see Sect. 4.2 for a description and Table 1 for timings). Horizontal dotted lines in $\theta_{B}, \phi_{B}$, and $\beta_{\mathrm{p}}$ mark values at $0^{\circ}, 180^{\circ}, 1$ as a reference, respectively. Color version available online.

Table 1. Timings (dd, hh:mm UT) of the interfaces for substructures inside the ICME, identified with numbered ticks in Figs. 1-4.

\begin{tabular}{lccc}
\hline \hline Tick number & Timing-ACE & Timing-Ulysses & Substructure \\
\hline 1 & $04,11: 00$ & $23,13: 30$ & sheath \\
2 & $04,14: 30$ & $23,22: 00$ & MC inbound \\
3 & $05,07: 45$ & $26,13: 00$ & MC outbound \\
4 & $05,20: 30$ & $28,00: 00$ & back \\
5 & $06,02: 30$ & $28,09: 00$ & \\
\hline
\end{tabular}

Earth toward the Sun, $\hat{\boldsymbol{y}}_{\mathrm{GSE}}$ is in the ecliptic plane and in the direction opposite to the planetary motion, and $\hat{z}_{\mathrm{GSE}}$ points to the north pole. However, Ulysses data are provided in the heliographic radial tangential normal (RTN) system of reference $(\hat{\boldsymbol{R}}$, $\hat{\boldsymbol{T}}, \hat{\boldsymbol{N}})$, in which $\hat{\boldsymbol{R}}$ points from the Sun to the spacecraft, $\hat{\boldsymbol{T}}$ the cross product of the Sun's rotation unit vector $(\hat{\boldsymbol{\Omega}})$ with $\hat{\boldsymbol{R}}$, and $\hat{\boldsymbol{N}}$ completes the righthanded system (e.g., Fränz \& Harper 2002).
To accurately compare the observations of vector quantities made from both spacecraft and the orientation of the flux rope at both locations, we rotated all the vectorial data from Ulysses to the local GSE system of ACE (when this spacecraft is at the closest approach distance of the MC axis). We describe this transformation of coordinates in Appendix A. This permits magnetic field components to be compared in the same frame (Figs. 1, 2), as well as the orientation of the MC at both positions.

We next define a local system of coordinates linked to the cloud (i.e., the cloud frame, Lepping et al. 1990) in order to better understand the cloud properties and to compare the results at both positions (such as the axial/azimuthal magnetic flux). The local axis direction of the MC defines $\hat{z}_{\text {cloud }}\left(\right.$ with $B_{z \text {,cloud }}>0$ ). Since the speed of the cloud is mainly in the Sun-Earth direction and is much higher than the spacecraft speed, which can be supposed to be at rest during the cloud's observing time, we assume a rectilinear spacecraft trajectory in the cloud frame. The trajectory defines a direction $\hat{\boldsymbol{d}}$, so we take $\hat{\boldsymbol{y}}_{\text {cloud }}$ in the direction $\hat{z}_{\text {cloud }} \times \hat{\boldsymbol{d}}$ and $\hat{\boldsymbol{x}}_{\text {cloud }}$ completes the right-handed orthonormal base $\left(\hat{\boldsymbol{x}}_{\text {cloud }}, \hat{\boldsymbol{y}}_{\text {cloud }}, \hat{\boldsymbol{z}}_{\text {cloud }}\right)$. Thus, $B_{x, \text { cloud }}, B_{y \text {,cloud }}$, and $B_{z, \text { cloud }}$ are the components of $\boldsymbol{B}$ in this new base.

The cloud frame is especially useful when the impact parameter, $p$ (the minimum distance from the spacecraft to the cloud 


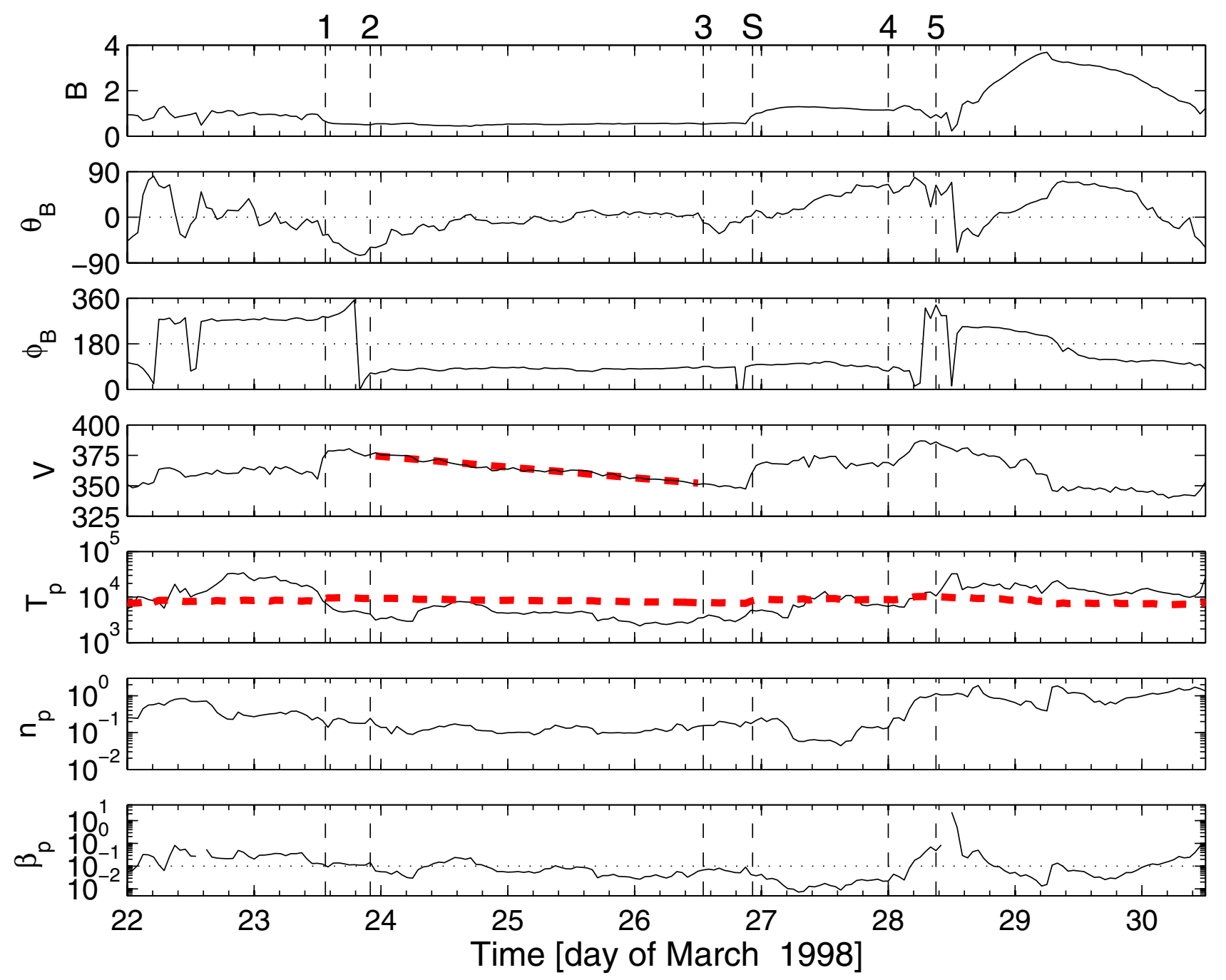

Fig. 2. In situ plasma and magnetic field parameters of the ICME observed in March 1998 by Ulysses located at 5.4 AU from the Sun. The format is the same as for Fig. 1. The magnetic field components are defined in the GSE frame on ACE (see Sect. 2.2). Vertical lines with the same reference number correspond to the same interfaces of substructures as at $1 \mathrm{AU}$. Color version available online.

axis), is small compared to the MC radius (called $R$ below). In particular, for $p=0$ and an MC described using a cylindrical magnetic configuration, $\boldsymbol{B}(r)=B_{z}(r) \hat{z}+B_{\phi}(r) \hat{\boldsymbol{\phi}}$, we have $\hat{\boldsymbol{x}}_{\text {cloud }}=\hat{\boldsymbol{r}}$ and $\hat{\boldsymbol{y}}_{\text {cloud }}=\hat{\boldsymbol{\phi}}$ after the spacecraft has crossed the MC axis. In this case and for a cylindrical flux rope, the magnetic field data obtained by the spacecraft will show: $B_{x, \text { cloud }}=0$, a large and coherent variation of $B_{y \text {,cloud }}$ (with a change of sign), and an intermediate and coherent variation of $B_{z \text {,cloud, from low }}$ values at one cloud edge, taking the highest value at its axis and returning to low values at the other edge $\left(B_{z, \text { cloud }}=0\right.$ is typically taken as the MC boundary).

One possible procedure for estimating the flux rope orientation is the classical minimum variance (MV) method applied to the normalized series of magnetic field measurements within the estimated boundaries of the MC (Sonnerup \& Cahill 1967). It was extensively used to estimate the orientation of MCs (see e.g., Lepping et al. 1990; Bothmer \& Schwenn 1998; Farrugia et al. 1999; Dasso et al. 2003; Gulisano et al. 2005), and it provides a good orientation estimation when $p$ is smaller than $R$ and if the in/out bound magnetic fields are not significantly asymmetric. Gulisano et al. (2007) have tested the MV using a static cylindrical Lundquist's solution. They find a deviation of the axis orientation from the model of typically $3^{\circ}$ for $p$ being $30 \%$ of $R$. This deviation remains below $20^{\circ}$ for $p$ as high as $90 \%$ of $R$. Another method of finding the MC orientation is called simultaneous fitting $(\mathrm{SF})$. It minimizes a residual function, which takes the distance into account between the observed time series of the magnetic field and a theoretical expression containing several free parameters, which include the angles for the flux rope orientation and some physical parameters associated with the physical model assumed for the magnetic configuration in the cloud (e.g., Hidalgo et al. 2002; Dasso et al. 2003).

\section{Modeling the magnetic cloud evolution}

\subsection{Self-similar expansion}

The evolution of an MC can be described with the model developed by Démoulin et al. (2008). Based on previous observations and theoretical considerations, a few basic hypothesis are introduced in this model. First, the MC dynamical evolution is split into two different motions: (i) a global one describing the position $\boldsymbol{r}_{\mathrm{CM}}(t)=D(t) \hat{\boldsymbol{v}}_{\mathrm{CM}}$ of the center of mass $(\mathrm{CM})$ with respect to a fixed heliospheric frame and (ii) an internal expansion where the elements of fluid are described with respect to the CM frame. 
Second, during the spacecraft crossing of the MC, the motion of the MC center is approximately a uniformly accelerated motion and thus

$D(t)=D_{0}+V_{0}\left(t-t_{0}\right)+a\left(t-t_{0}\right)^{2} / 2$.

Third, the cloud coordinate system $\left(\hat{\boldsymbol{x}}_{\text {cloud }}, \hat{\boldsymbol{y}}_{\text {cloud }}, \hat{\boldsymbol{z}}_{\text {cloud }}\right)$ defines the three principal directions of expansion. Fourth, the expansion of the flux rope is self-similar with different expansion rates in each of the three cloud's main axes. In the CM frame, this assumption implies that the position, $\boldsymbol{r}(t)$, of a element of fluid is described by

$$
\begin{aligned}
\boldsymbol{r}(t) & =x(t) \hat{\boldsymbol{x}}_{\text {cloud }}+y(t) \hat{\boldsymbol{y}}_{\text {cloud }}+z(t) \hat{\boldsymbol{z}}_{\text {cloud }} \\
& =x_{0} e(t) \hat{\boldsymbol{x}}_{\text {cloud }}+y_{0} f(t) \hat{\boldsymbol{y}}_{\text {cloud }}+z_{0} g(t) \hat{z}_{\text {cloud }},
\end{aligned}
$$

where $x(t), y(t)$, and $z(t)$ are the fluid coordinates from the CM reference point at time $t$, and where $x_{0}, y_{0}$, and $z_{0}$ are the position coordinates taken at a reference time $t_{0}$. The time functions $e(t), f(t)$, and $g(t)$, provide the specific time functions for the self-similar evolution. Finally, based on observations of different MCs at different distances from the Sun (e.g., Liu et al. 2005; Wang et al. 2005a; Leitner et al. 2007; Gulisano et al. 2010), we approximate $e(t)$ by the function

$e(t)=\left(D(t) / D_{0}\right)^{l}$

and similar expressions for $f(t)$ and $g(t)$, simply replacing the exponent $l$ by $m$ and $n$, respectively, in order to permit an anisotropic expansion.

From the conservation of mass we model the decay of the proton density as

$n_{\mathrm{p}}=n_{\mathrm{p}, 0}\left(D / D_{0}\right)^{-(l+m+n)}$.

From the kinematic self-similar expansion proposed before, and assuming an ideal evolution (i.e., non-dissipative, so that the magnetic flux across any material surface is conserved), the evolution of the magnetic components advected by the fluid is

$$
\begin{aligned}
& B_{x, \text { cloud }}=B_{x, \text { cloud }_{0}}\left(D / D_{0}\right)^{-(m+n)}, \\
& B_{y, \text { cloud }}=B_{y, \text { cloud }_{0}}\left(D / D_{0}\right)^{-(l+n)}, \\
& B_{z, \text { cloud }}=B_{z, \text { loud }_{0}}\left(D / D_{0}\right)^{-(l+m)} .
\end{aligned}
$$

With the above hypothesis and neglecting the evolution of the spacecraft position during the MC observation, the observed velocity profile $\left(V_{x}\right)$ along the direction $\hat{\boldsymbol{v}}_{\mathrm{CM}}$ of the center of mass velocity is expected to be (Démoulin et al. 2008)

$$
\begin{aligned}
V_{x} & =-V_{0}-a\left(t-t_{0}\right)+V_{0} \frac{t-t_{0}}{D_{0} / V_{0}+t-t_{0}} \zeta \\
& \approx-V_{0}+\frac{V_{0}^{2}}{D_{0}} \zeta\left(t-t_{0}\right),
\end{aligned}
$$

where $\gamma$ is the angle between $\hat{z}_{\text {cloud }}$ and $\hat{\boldsymbol{v}}_{\mathrm{CM}}$, and

$$
\zeta=l \sin ^{2} \gamma+n \cos ^{2} \gamma
$$

For typical values of MCs we can linearize Eq. (7) in $t-t_{0}$ and neglect the acceleration $a$ (Démoulin et al. 2008). This implies that the slope of the observed linear velocity profile provides information on the expansion rate of the flux rope in the two combined directions: $\hat{\boldsymbol{x}}_{\text {cloud }}$ and $\hat{\boldsymbol{z}}_{\text {cloud }}$ (since $\zeta$ involves both $l$ and $n)$.

\subsection{Magnetic field}

Since MCs have low plasma $\beta$ (a state near to a force-free field) and present flux rope signatures, its magnetic configuration is generally modeled using the cylindrical linear force-free field $\boldsymbol{B}^{\mathrm{L}}=B_{0}\left[J_{1}\left(\alpha_{0} r\right) \hat{\boldsymbol{\phi}}+J_{0}\left(\alpha_{0} r\right) \hat{z}\right]$ (Lundquist 1950). If the expansion coefficients in the three main cloud's axes $(l, m, n)$ were significantly different, then an initial Lundquist configuration would be strongly deformed. However, observations of the MC field configuration are approximately consistent with this magnetic configuration at different heliodistances, ranging from 0.3 to $5 \mathrm{AU}$ (e.g., Bothmer \& Schwenn 1998; Leitner et al. 2007), so that we expect a small anisotropy on the expansion along different cloud directions (i.e., $l \approx m \approx n$ ). From observations of different MCs at significantly different heliodistances (Wang et al. 2005b; Leitner et al. 2007) and from observations of the velocity profile slope from single satellite observations (Démoulin et al. 2008; Gulisano et al. 2010), it has been found that $\zeta \approx 0.8$. Since $\zeta$ is a combination of $l$ and $n$, which depends on $\gamma$ for each cloud, a systematic difference on $l$ and $n$ would be detected in a set of MCs with variable $\gamma$ angle. Such a systematic variation of $l$ and $n$ with $\gamma$ was not found.

The kinematic self-similar expansion given in the previous section combined with a non-dissipative regime, as expected for space plasmas, provide a prediction for the observed magnetic configuration during the transit of the MC. Then, assuming an initial Lundquist configuration, the observations are modeled as (Démoulin et al. 2008)

$$
\begin{aligned}
& B_{x, \text { cloud }}(t)=-\frac{p}{\rho(t)} \frac{B_{0}}{f(t) g(t)} J_{1}[U(t)], \\
& B_{y, \text { cloud }}(t)=\frac{V_{\mathrm{c}}\left(t-t_{\mathrm{c}}\right) \sin \gamma}{\rho(t)} \frac{B_{0}}{e(t) g(t)} J_{1}[U(t)], \\
& B_{z, \text { cloud }}(t)=\frac{B_{0}}{e(t) f(t)} J_{0}[U(t)],
\end{aligned}
$$

where $U(t)=\frac{\alpha_{0} \rho(t)}{\sqrt{e(t)^{2}+f(t)^{2}}}, \rho(t)=\sqrt{\left(V_{\mathrm{c}}\left(t-t_{\mathrm{c}}\right) \sin \gamma\right)^{2}+p^{2}}, B_{0}$ is the strength of the magnetic field, and $\alpha_{0} / 2$ is the twist of the magnetic field lines near the center, at time $t=t_{\mathrm{c}}$. By construction of the self-similar expansion, this magnetic field is divergence-free at any time.

Equations (10)-(12) have free parameters that are computed by fitting these equations to in situ observations, by minimizing a residual function and quantifying the square of the difference between the observed and the predicted values for the magnetic field components (i.e., a least square fit). This provides information on the observed flux rope, such as its orientation, its extension, and its magnetic flux. We call this method the expansion fitting (EF) method, and EFI method when isotropy $(l=m=n)$ is assumed.

\subsection{Global magnetic quantities using the Lundquist model}

Quantification of global magnetic quantities, such as the socalled ideal-MHD invariants, has been very useful for comparing and associating MCs and their solar sources (see, e.g., Mandrini et al. 2005; Dasso et al. 2005b; Dasso 2009). These MHD invariants are computed using a specific model of the MC magnetic configuration (Sect. 3.2).

For the Lundquist's solution, the axial flux is

$F_{z, \text { Lund }}=2 \pi \int_{0}^{R} B_{z} r \mathrm{~d} r=2 \pi J_{1}\left(\alpha_{0} R_{0}\right) \frac{B_{0} R_{0}}{\alpha_{0}}$, 
where $R_{0}$ is the flux rope radius at a reference time $t_{0}$. The azimuthal flux is

$$
F_{\varphi, \text { Lund }}=L \int_{0}^{R} B_{\varphi} \mathrm{d} r=\left(1-J_{0}\left(\alpha_{0} R_{0}\right)\right) \frac{B_{0} L_{0}}{\alpha_{0}},
$$

where $L_{0}$ is the axial length of the flux rope at $t_{0}$. For a flux rope staying rooted to the Sun, $L(t)$ is typically close to the distance to the Sun $D(t)$.

The relative magnetic helicity is (Dasso et al. 2003; Nakwacki et al. 2008b)

$$
\begin{aligned}
H_{\text {Lund }} & =4 \pi L \int_{0}^{R} A_{\varphi} B_{\varphi} r \mathrm{~d} r \\
& =2 \pi\left(J_{0}^{2}+J_{1}^{2}-\frac{2 J_{0} J_{1}}{\alpha_{0} R_{0}}\right) \frac{L_{0} B_{0}^{2} R_{0}^{2}}{\alpha_{0}} .
\end{aligned}
$$

The magnetic energy content is not an invariant in MCs. To compute its decay rate, we simplify and assume that the MC expansion is isotropic with $e(t)=f(t)=g(t)$. Then, the magnetic energy ( $\left.E_{\text {Lund }}\right)$ is computed as (Nakwacki et al. 2008b)

$$
\begin{aligned}
E_{\text {Lund }}(t) & =\frac{2 \pi L}{2 \mu_{0}} \int_{0}^{R} B^{2} r \mathrm{~d} r \\
& =\frac{\pi}{e(t)}\left(J_{0}^{2}+J_{1}^{2}-\frac{J_{0} J_{1}}{\alpha_{0} R_{0}}\right) \frac{L_{0} B_{0}^{2} R_{0}^{2}}{\mu_{0}}=\frac{E_{\text {Lund }}\left(t_{0}\right)}{e(t)},
\end{aligned}
$$

where $\mu_{0}$ is the magnetic permeability. Thus, for the isotropic expansion, the magnetic energy decays with time as $e(t)^{-1}$. In the case of a small anisotropic expansion, a similar decay is expected.

\subsection{Global magnetic quantities using the direct method}

We define below a method of estimating the global magnetic quantities directly from the observations. This method assumes first that the cross section is circular, second that there is symmetry of translation of $\boldsymbol{B}$ along the main axis of the flux rope, and finally that the impact parameter is low (so $B_{y} \approx \pm B_{\phi}$ and $r \approx x$ ). We also separate the time range covered by the cloud in two branches (the inbound/outbound branches corresponding to the data before/after the closest approach distance to the flux rope axis, see e.g., Dasso et al. 2005a), and we consider each branch separately. We call this method DM-in/DM-out, depending on the branch (inbound/outbound) that is used. We define the accumulative magnetic fluxes for the axial and azimuthal field components, in the inbound branch as

$$
\begin{aligned}
& F_{z, \mathrm{DM}-\mathrm{in}}(x)=2 \pi \int_{X_{\mathrm{in}}}^{x} B_{z}\left(x^{\prime}\right) x^{\prime} \mathrm{d} x^{\prime} \\
& F_{y, \mathrm{DM}-\mathrm{in}}(x)=L \int_{X_{\mathrm{in}}}^{x} B_{y}\left(x^{\prime}\right) \mathrm{d} x^{\prime},
\end{aligned}
$$

where $X_{\text {in }}$ is the $x$ value at the starting point of the flux rope and $\mathrm{d} x=V_{x} \mathrm{~d} t$. Next, from $B_{y}(x)$ and $F_{z, \mathrm{DM}-\text { in }}(x)$ we obtain an expression for the magnetic helicity (Dasso et al. 2006)

$H_{\mathrm{DM}-\text { in }}=2 L \int_{X_{\text {in }}}^{X_{\text {center }}} F_{z, \text { DM-in }}(x) B_{y}(x) x \mathrm{~d} x$.

Finally, the magnetic energy is computed from the direct observations of $B(t)=|\boldsymbol{B}(t)|$,

$E_{\mathrm{DM}-\mathrm{in}}=\frac{\pi L}{\mu_{0}} \int_{X_{\mathrm{in}}}^{X_{\text {center }}} B^{2}(x) x \mathrm{~d} x$.
In an equivalent way, we define the same quantities for the outbound branch. These equations are used to estimate these global quantities directly from in situ observations of the magnetic field, using the components of the field and the integration variable $(x)$ in the local MC frame.

\section{Results for the studied MC}

\subsection{Common frame for data from ACE and Ulysses}

We first describe the in situ data of both spacecraft in GSE coordinates (defined at the time of ACE observations, see Appendix A). Figure 1 shows the magnetic field and plasma ACE observations. The flux rope extends from ticks " 2 " to " 4 ". There, the magnetic field is stronger by a factor $\approx 3$ than in the surrounding SW, and it has a coherent rotation. At ACE position the velocity profile is almost linear within the MC (Fig. 1). The density is relatively high, up to $\approx 20 \mathrm{~cm}^{-3}$, compared to the density present in the $\mathrm{SW}$ before the MC sheath, $\approx 5 \mathrm{~cm}^{-3}$. In most of the MC the proton temperature is clearly lower than the expected temperature in a mean SW with the same speed (Lopez \& Freeman 1986; Elliott et al. 2005; Démoulin 2009); this is a classical property of MCs.

Figure 2 shows Ulysses observations, in the same format as Fig. 1. At the Ulysses position, the linear velocity profile is still present before a strong shock on March 26, 22:20 UT (tick "S" in Fig. 2). The proton temperature is, as typically observed in MCs, well below the temperature expected for a typical SW at 5.4 AU from an extrapolation of the empirical law given by Lopez \& Freeman (1986). Unlike to ACE, the density is much lower in the MC than in the SW present before the MC sheath $\left(\approx 0.1-0.2 \mathrm{~cm}^{-3}\right.$ in the MC compared to $\approx 0.5-1 \mathrm{~cm}^{-3}$ in the $\mathrm{SW})$. With mass conservation, this implies that the volume expansion rate of the MC is much higher than the SW one. The magnetic field observed on Ulysses, has very significantly decreased with respect to the field on ACE (factor $\approx 20$ ), becoming even weaker than the one present in the surrounding SW (typically by a factor $\approx 2$ ). This is consistent with the strong observed decrease in density.

\subsection{Magnetic cloud on ACE}

The definition of the MC boundaries is an important step in the analysis of an MC since the selected boundaries are affecting all the physical quantities related to the magnetic field. The MC boundaries are associated to discontinuities in the magnetic field because such discontinuities are formed in general at the boundary of two regions having different magnetic connectivities, such as the flux rope and its surrounding medium magnetically linked to the SW.

The front boundary of MCs is typically better defined than the back (or rear) boundary. Such is the case in the analyzed MC on ACE, where there is a fast forward shock at tick " 1 ", and a strong discontinuity of $\phi_{B}$ (at tick " 2 " in Fig. 1). Moreover, the magnetic field in front has a reverse $\phi_{B}$ and, between ticks " 1 " and " 2 ", $\boldsymbol{B}$ is fluctuating, a characteristic of MC sheaths. The density also has a discontinuity and is enhanced just before tick " 2 ", another characteristic of MC sheaths. Then, the MC front boundary is set at tick " 2 ". It is worth noting that the shock at tick " 1 " was previously identified in Wind spacecraft data at 11:05 UT as an ICME related shock (see http://pwg. gsfc.nasa.gov/wind/current_listIPS.htm). This interplanetary fast forward shock-wave has been recently studied in a multi-spacecraft analysis by Koval \& Szabo (2010). For further 

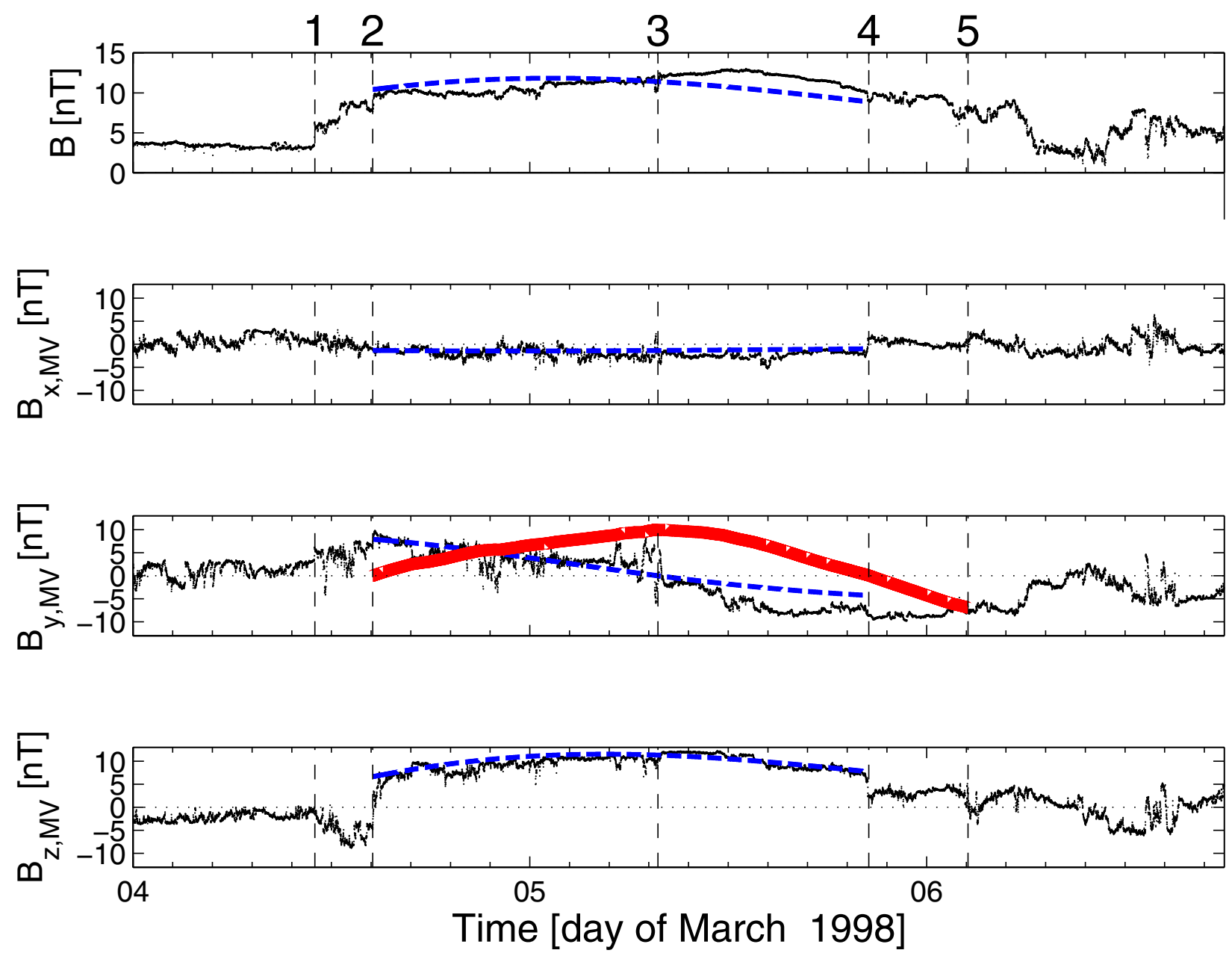

Fig. 3. Strength and components of the magnetic field vector at $1 \mathrm{AU}$ in the cloud frame given by the MV method (see Sect. 4.2). Numbered ticks are the same as in Fig. 1. The dashed black lines are the Lundquist field model fitted with the EFI method (Sect. 3.2). The black thick line is the accumulative magnetic flux for the azimuthal component (in arbitrary units). Color version available online.

details on the characteristics of shocks and their identification in the interplanetary medium, see Vinas \& Scudder (1986) and Berdichevsky et al. (2000).

The back boundary is also set at a discontinuity of the magnetic field. As is typical in MCs, there are several possibilities after 18 UT on March 5 (see Fig. 1). However, $\phi_{B}$ and $\theta_{B}$ have clear discontinuities, similar but weaker than the front one, at tick "4". This is confirmed by a discontinuity in the density. The above boundaries are used to find the orientation of the flux rope both with the MV and the SF (Sect. 2.2). Indeed, the MC boundaries are better defined in the MC frame since the axial and azimuthal field components are separated (Fig. 3), so we need to determine the MC frame.

We first use the MV method to find the direction of the MC axis. The MV should be applied only to the flux rope, otherwise if part of the back is taken into account the directions given by the MV could be significantly bias (the back region is no longer part of the flux rope at the observation time, Gulisano et al. 2007). Then, an iteration is needed starting with the first estimation of the flux-rope boundaries from the data, performing the MV analysis, then plotting the magnetic field in the cloud coordinates, and finally checking if the selected back boundary is correct with the accumulated azimuthal flux (Eq. (19)). For the studied MC on ACE, the orientation found with the MV provides an almost vanishing accumulated flux at the back boundary selected above (Fig. 3). Then, no iteration is needed, and the
Table 2. Magnetic cloud orientation according to minimum variance and simultaneous fitting.

\begin{tabular}{lccc}
\hline \hline Model & Parameter & ACE & Ulysses \\
\hline Min. Var & $\theta$ & $12^{\circ}$ & $-29^{\circ}$ \\
Sim. Fit & $\theta$ & $-11^{\circ}$ & $-14^{\circ}$ \\
\hline Min. Var & $\phi$ & $101^{\circ}$ & $84^{\circ}$ \\
Sim. Fit & $\phi$ & $115^{\circ}$ & $81^{\circ}$ \\
\hline
\end{tabular}

MV provides dependable orientation within the accuracy of the method (Table 2 ). The low mean value of $B_{x, \text { cloud }}$ indicates that the impact parameter is small (Fig. 3). This implies that the orientation found by the MV method could differ from the real one by typically $10^{\circ}$ (Gulisano et al. 2007).

The MC axis direction is also estimated with a standard simultaneous fit (SF) of the Lundquist solution to the observations (see end of Sect. 2.2). The fitting minimizes the distance of the model to the observed magnetic field in a least square manner. As for the MV, it is important to take only the data into account in the range where the flux rope is present. The output of the SF method provides estimations for the MC frame vectors as the $\mathrm{MV}$, and also the impact parameter and the physical parameters (the free parameters of the Lundquist's solution). There is a significant difference in the MC axis orientations between the MV 
and the SF methods, larger in latitude $\left(23^{\circ}\right)$ than in longitude $\left(14^{\circ}\right)$. Moreover, with the SF results the accumulated azimuthal flux is significant at the back boundary (so the magnetic flux is not balanced), and there are no nearby significant discontinuities. We conclude that the MV method provides a better approximation of the MC frame than the SF method in this particular MC. Since $\phi$ is close to $90^{\circ}$, it is a good assumption to consider that $\mathrm{ACE}$ crosses the flux rope front (or nose). Moreover, since $\theta$ is small, the MC axis lies almost in the ecliptic plane.

In summary, on ACE we select the MC boundaries as March 4, 1998, at 14:30 UT and March 5, 1998, at 20:30. The front boundary differs by up to $\approx 5 \mathrm{~h}$ from previous studies. The larger difference is with the back boundary of the MC since it was set on March 6 at 03:15 (Skoug et al. 2000), 2:30 UT (Liu et al. 2005), and 06:30 UT (Du et al. 2007), so significantly later than our boundary set at tick " 4 ". Our boundaries take the extension of the flux rope into account when it was crossing ACE. In fact, part of the MC characteristics are present after the back boundary "4" (e.g., strong and relatively coherent magnetic field, cooler temperature than expected), but some other quantities are closer to typical SW values, such as $\beta_{\mathrm{p}}$ which is more than 1 . This was previously found in other MCs (Dasso et al. 2006, 2007), and was called a back region. This type of region was interpreted as formed by a magnetic field and plasma belonging to the flux rope when it was close to the Sun, and later connected to the SW due to magnetic reconnection in the front of the flux rope, which in a low-density plasma like the SW could be more efficient due to the Hall effect (e.g., Morales et al. 2005), so a back region has intermediate properties between MC and SW since it is a mixture of the two. Indeed, this is the case at the time of ACE observations since $B_{z \text {,cloud }}$ is fluctuating in the back region, while $B_{y, \text { cloud }}$ retains its coherency more as in the flux rope (Fig. 3).

\subsection{Magnetic cloud at the position of Ulysses}

On Ulysses the MC has a complex structure in most of the measured parameters. The proton temperature is significantly below the expected temperature in the interval of time between tick " 1 " and about one day after tick " 3 " (Fig. 2). At the beginning of this time interval, the magnetic field has a coherent rotation, then later it is nearly constant up to a large discontinuity of the field strength (at tick "S", $\approx 0.4$ days after tick "3"). Later, the magnetic field is stronger with a significant rotation. Then, the observations on Ulysses show a flux rope with characteristics significantly different from a "standard" flux rope such as observed on ACE. A magnetic field strength flatter than on ACE is expected if the flux rope transverse size increases much less rapidly than its length, since it implies that the axial component becomes dominant with increasing distance to the Sun so that the magnetic tension becomes relatively weaker in the force balance (Démoulin \& Dasso 2009a). The strong discontinuity followed by a strong field is more peculiar. Indeed, this MC was strongly overtaken by a faster structure, as shown by the velocity panel of Fig. 2, and previously identified by Skoug et al. (2000).

The proton temperature has a similar variation for both spacecraft since it becomes lower than the expected temperature approximately after the shock defined by the discontinuity of $V$. This discontinuity defines the position of tick "1" (Fig. 2). The front boundary of the MC is defined less well than on ACE since there is no discontinuity. Nevertheless, the behavior of the magnetic field is similar at the position of both spacecraft as follows. Before tick " 2 ", $\theta_{B}$ is fluctuating while globally decreasing, while after tick " 2 " it gradually increases (with fluctuations) on both spacecraft. The value of $\phi_{B}$ shows a global behavior similar to a step function on both spacecraft, with nearly constant values both before tick " 1 " and after tick " 2 ". The main difference for $\phi_{B}$ is a discontinuity at tick " 2 " at $1 \mathrm{AU}$, while $\phi_{B}$ has a smooth transition at 5.4 AU. Then, we fix the beginning of the MC, tick " 2 ", at the beginning of the period where $\theta_{B}$ starts increasing and $\phi_{B}$ is nearly constant. This is confirmed in the MC frame, as $B_{z, \mathrm{MV}} \approx 0$ at tick " 2 " at both 1 and $5.4 \mathrm{AU}$ (Figs. 3, 4).

The back boundary is much more difficult to define since there are several possibilities and the absence of a clear coherence between all the measured parameters. We tried several back boundaries, the MV and the SF methods, and used the iterative method described in Sect. 4.2 to check the selected boundary in the derived MC cloud frame. We found that the MC axis orientation can change by more than $20^{\circ}$ and that the selected boundaries are not confirmed by the cancellation of the accumulated flux.

The difficulties of both the MV and the SF methods stem from the large asymmetry of the MC when observed at the position of Ulysses spacecraft. Even normalizing the magnetic field strength is not sufficient since the outbound branch is strongly distorted after the shock (tick " $\mathrm{S}$ "). This is the consequence of the overtaking flow seen after tick " 5 " in Fig. 2. The MC observations have comparable characteristics to the MHD simulations of Xiong et al. $(2007,2009)$ where they modeled the interaction of a flux rope with another faster one. On Ulysses, the internal shock has propagated nearly up to the MC center, so that most of the outbound branch is strongly distorted. This implies that both the MV and the SF methods cannot be used to find the MC orientation on Ulysses.

In the MC frame deduced from ACE, the magnetic field components measured on Ulysses between ticks " 2 " and " 3 " have the expected behavior for a flux rope (Fig. 4), as follows. The value of $B_{x, \text { cloud }}$ is almost constant and small, indicating a low-impact parameter. $B_{y, \text { cloud }}$ shows a clear rotation and $B_{z \text {,cloud }}$ increases from ticks " 2 " to " 3 ". These are indications that the studied MC has not significantly changed its orientation $\left(\leq 10^{\circ}\right)$ between 1 and 5.4 AU, despite the SW overtaking the rear region of the flux rope. Therefore, for Ulysses we use below the MC frame found from ACE.

The accumulated azimuthal flux is at its maximum after the internal shock position (Fig. 4), an indication that the shock would have overtaken the flux rope center. However, we cannot trust the behavior of the accumulated azimuthal flux behind the shock since the orientation and the strength of the magnetic field are strongly modified. Indeed, all the magnetic field components are perturbed even before the shock, in the interval ["3","S"]. Before tick " 3 ", $B_{y \text {,cloud }}$ is weak, and it almost vanishes at tick " 3 ", while $B_{z \text {,cloud }}$ is nearly maximum there, so we set the flux rope center at tick " 3 ".

In summary, on Ulysses we initially select the ICME boundaries, including the sheath, as from March 23 at 13:30 UT (tick "1") to March 28 at 09:00 UT (tick "5"). The boundaries chosen by Skoug et al. (2000) were slightly different since they chose the range from March 24 at 02:00 UT to March 28 at 02:30 UT. The analysis of the magnetic field behavior above indicates that the inbound branch of the $\mathrm{MC}$ is from tick " 2 " to " 3 ", with an outbound branch from " 3 " to "4" that is strongly distorted. The inbound extension is confirmed by a similar behavior of the velocity and the proton temperature at 1 and $5.4 \mathrm{AU}$. 

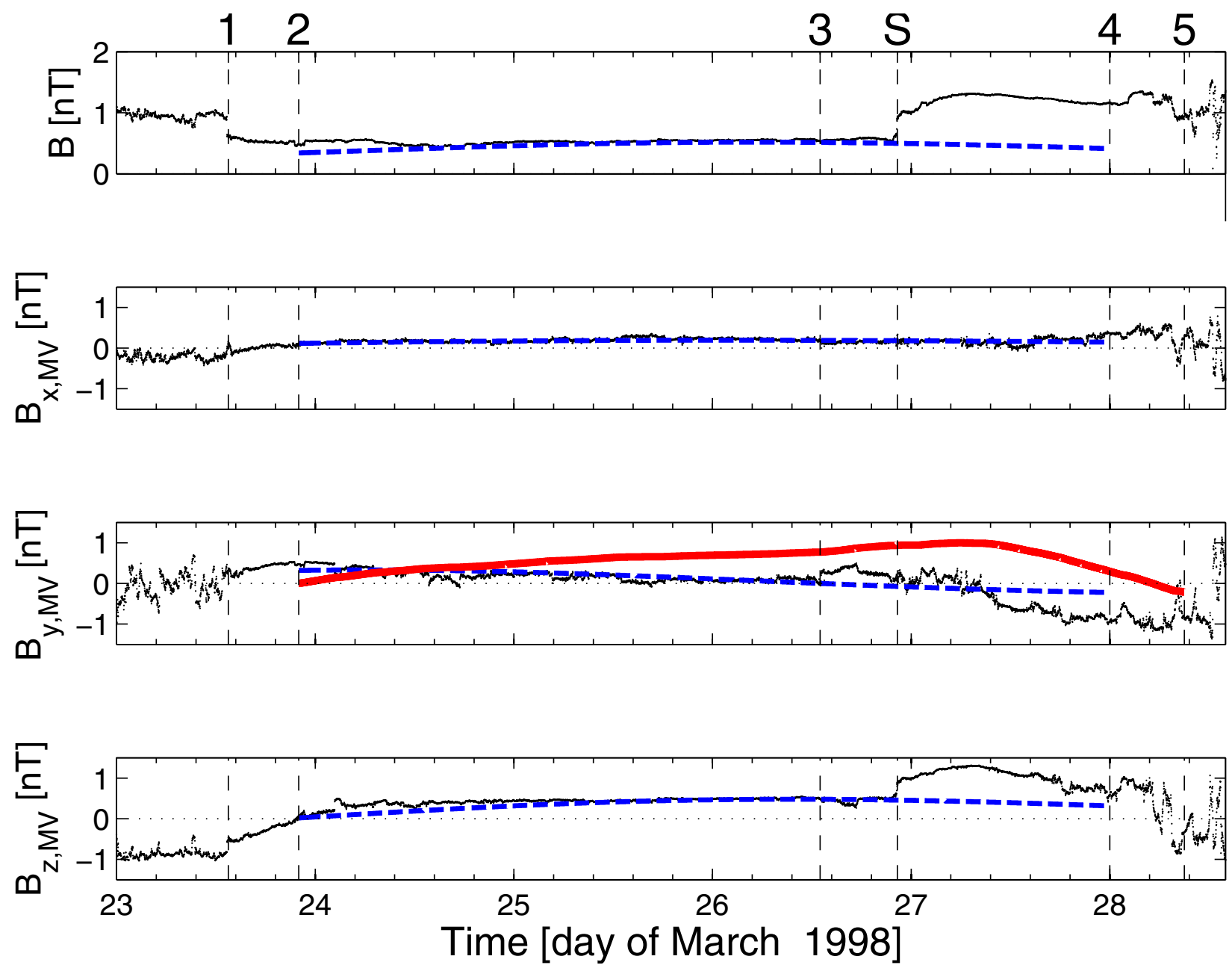

Fig. 4. Strength and components of the magnetic field vector at 5.4 AU in the cloud frame given by the MV method applied at 1 AU on ACE data (see Sect. 4.3). The format is the same as for Fig. 3. Color version available online.

\subsection{Impact parameter from ACE and Ulysses}

The agreement between the characteristics of the MC observed from ACE and Ulysses are clear indications that the same MC was observed. In this section we estimate the impact parameter when the MC is observed at each of the two spacecraft.

Figure 3 shows that $B_{x, \text { cloud }}$ is small compared to $B$, as expected when the impact parameter $(p)$ is close to zero. From the mean value of $B_{x, \text { cloud }}$, we estimate $p / R \approx 0.3$ (see the method in Gulisano et al. 2007). From the inbound size $S_{\mathrm{ACE}, \text { in }}=0.144 \mathrm{AU}$ and $p / R=0.3$, we estimate that the radius $R_{\mathrm{ACE}} \approx 0.15 \mathrm{AU}$, with a circular cross-section. Fitting the value of $p / R$, using the SFEFI method, we also obtain $p / R \approx 0.3$. Another estimation of the impact parameter can be done using the approximation introduced in the simplest form of Eq. (31), $\left\langle B_{x, \text { cloud }}\right\rangle /\langle B\rangle \approx 1.2 p / R$ (Démoulin \& Dasso 2009b), assuming a cross section roughly circular. We obtain $p / R \approx 0.27$ from this method. Thus, in conclusion, the impact parameter when ACE observes the cloud is low $(p / R \approx 0.3)$ and very similar to its estimation using different proxies.

From the mean value of $B_{x, \text { cloud }}$ on Ulysses (see Fig. 4), as done for ACE, we estimate $p / R=0.54$. From $S_{\mathrm{Uly}, \text { in }}=0.55 \mathrm{AU}$ and $p / R=0.54$, we estimate that $R_{\mathrm{Uly}} \approx 0.65$ AU. The above values of $R$ on both spacecraft agree with the values found from fitting the free parameters of the cylindrical expansion Lundquist model (EFI method, see Sect. 3.2, Eqs. (10)-(12)). Again, using also the estimation of the impact parameter from the simplest approximation of Démoulin \& Dasso (2009b) $\left(\left\langle B_{x, \text { cloud }}\right\rangle /\langle B\rangle \approx\right.$ $1.2 p / R)$, we obtain $p / R \approx 0.18$ from this method. Thus, in conclusion, the impact parameter when Ulysses observes the cloud is also low, with $p / R$ in the range [0.2-0.5] using different proxies.

Further arguments in favor of the association of the $\mathrm{MC}$ observed on ACE and Ulysses are given in the next two sections with the timing and the agreement of the mean velocity, expansion rate, magnetic fluxes, and helicity as deduced at the two locations.

\subsection{Acceleration and expansion}

The translation velocity of the flux rope on ACE is estimated, as typically done, as the mean value of the observed bulk speed during the observation range ["2", "4"]. It turns out to be $V_{\mathrm{ACE}}=-348 \mathrm{~km} \mathrm{~s}^{-1}$. However, for Ulysses, because of the perturbation on the outbound branch, it is not possible to apply this classical procedure. Then, we computed a mean value of the observed speed only in a symmetric range near the center (tick " 3 "). We choose a range of $12 \mathrm{~h}$ around " 3 ", which gives $V_{\text {Ulysses }}=-351 \mathrm{~km} \mathrm{~s}^{-1}$, so that on Ulysses, the MC travels slightly faster than on ACE, with a mean acceleration of $\langle a\rangle=\left|V_{\text {Ulysses }}-V_{\mathrm{ACE}}\right| / \Delta t=0.14 \mathrm{~km} \mathrm{~s}^{-1} / \mathrm{d}$, where $\Delta t=21.2$ days is the elapsed time between both centers. However, the low value obtained for the acceleration just indicates that it was almost 
negligible during the transit from ACE to Ulysses, and it implies a negligible contribution to the interpretation of the observed velocity profile as a proxy of the expansion of the flux rope (Eq. (7), see Démoulin et al. 2008, for a justification). Then, we consider below that the MC is traveling from ACE to Ulysses with a constant velocity.

We fit the velocity observations to the velocity model described in Sect. 3.1 (Eq. (8)). From the fitted curve (panel 4, Figs. 1 and 2), we obtain the expansion coefficients $\zeta_{\mathrm{ACE}}=0.74$ for ACE and $\zeta_{\text {Uly }}=0.67$ for Ulysses. These values are very similar at both heliodistances, and they are consistent with previous results found at $1 \mathrm{AU}$ with the same method for a set of $26 \mathrm{MCs}$ (Démoulin et al. 2008), as well as the results obtained from a statistical analysis of MCs or ICMEs observed at various distances of the Sun (Bothmer \& Schwenn 1994; Chen 1996; Liu et al. 2005; Leitner et al. 2007).

The angle, $\gamma$, between the axis of the MC and the direction of motion defines the contribution of the axial and ortho-radial expansion rate to the observed $v_{x}(t)$, see Eq. (9). From the orientation given by $\mathrm{MV}$ on $\mathrm{ACE}$, this angle is $\gamma=101^{\circ}$. This implies that the expansion rate measured from $v_{x}$ is mainly due to the expansion in a direction perpendicular to the cloud axis and, then, for the cloud analyzed here $\zeta \approx l$.

The value of $\zeta$ observed on a given spacecraft, just provides the "local" expansion rate, which corresponds to the expansion during the in situ observations. However, since $\zeta_{\text {ACE }} \approx \zeta_{\text {Uly }}$, we assume that during the full travel between ACE and Ulysses the mean expansion rate occurred at a value between $\zeta=0.67$ and $\zeta=0.74$.

Assuming a self-similar expansion in the Sun-spacecraft direction (as in Eq. (4)), we can link (without any assumptions on the cloud shape and symmetries for the flux rope) the size of the structure along the direction of the MC motion $\left(\hat{\boldsymbol{v}}_{\mathrm{CM}}\right)$, at both heliodistances with the expected size on Ulysses given by $S_{\mathrm{ULY}, \exp }=S_{\mathrm{ACE}, \mathrm{obs}}(5.4)^{\zeta}$.

Because the outbound branch of the MC on Ulysses is strongly distorted, we analyze the inbound branch. Then, using the mean velocity and the time duration between ticks " 2 " and "3", we find that $S_{\mathrm{ACE}, \text { in }}=0.144 \mathrm{AU}$. Then, from the observed value of $\zeta_{\mathrm{ACE}}$, we find an expected size on Ulysses of $S_{\text {Uly,exp-in }}=0.498$ AU (using the observed $\zeta_{\text {Uly }}$, we obtain $\left.S_{\text {Uly,exp-in }}^{\prime}=0.444 \mathrm{AU}\right)$. This implies an expected center time on March 26 at 9:00 UT (and even an earlier time when using $\zeta_{\text {Uly }}$ ). However, the cumulative azimuthal flux ( $F_{y}$, Fig. 4 panel 3 ) smoothly and monotonically increases beyond this time, with a strong discontinuity of $B_{y}$ a bit later, on March 26, 13:00 UT. At the center of the flux rope we expect a global extreme of $F_{y}$ (Dasso et al. 2006, 2007; Gulisano et al. 2010), in particular, as it was observed when this same cloud was located at $1 \mathrm{AU}$ (panel 3 of Fig. 3). However, it is not the case in Ulysses at the expected time (9:00 UT), so that we decide to take the center at 13:00 UT, the time when $B_{y}$ started to be disturbed. This lack of exact agreement between the predicted and observed center positions could be associated with the inexact alignment between ACE and Ulysses.

\subsection{Prediction of the mean plasma density and magnetic field on Ulysses}

The expected values of the proton density and magnetic field on Ulysses can be predicted using the observations from ACE and the expansion rates in the three directions $(l, m$, and $n$, see Eqs. (5), (6)). The mean expansion rate, $\zeta$, along the plasma flow
Table 3. Mean values of proton density and magnetic field.

\begin{tabular}{llllll}
\hline \hline Quantity & ACE & Uly & UlyP1 & UlyP2 & UlyP3 \\
\hline$l$ & & & 0.7 & 0.7 & 0.74 \\
$m$ & & & 0.7 & 1. & 1. \\
\hline$n p\left(\mathrm{~cm}^{-3}\right)$ & 15.8 & 0.12 & $0.27(2.2)$ & $0.17(1.4)$ & $0.16(1.3)$ \\
$B(\mathrm{nT})$ & 10.3 & 0.48 & $0.88(1.7)$ & $0.56(1.1)$ & $0.53(1.0)$ \\
$B_{y, \mathrm{MV}}(\mathrm{nT})$ & 4.1 & 0.18 & $0.51(2.8)$ & $0.24(1.3)$ & $0.22(1.2)$ \\
$B_{z, \mathrm{MV}}(\mathrm{nT})$ & 9.0 & 0.42 & $0.85(2.0)$ & $0.51(1.2)$ & $0.48(1.2)$ \\
\hline
\end{tabular}

Notes. Observed (ACE and Ulysses) mean values of proton density $\left(n_{\mathrm{p}}\right)$ and magnetic field $\left(B, B_{y, \mathrm{MV}}\right.$, and $\left.B_{z, \mathrm{MV}}\right)$ for the inbound branch of the magnetic cloud, for three different estimations of expansion rates (see Sect. 4.6). The predicted to observed ratio, on Ulysses, is shown in parenthesis.

can be estimated with a value between $\zeta_{\mathrm{ACE}}$ and $\zeta_{\text {Uly }}$, which results in $l \approx \zeta$ for the orientation of this cloud. The presence of bi-directional electrons supports the connectivity of this MC to the Sun (Skoug et al. 2000); then, the axial expansion rate is estimated as $n \approx 1$, because the axial length needs to evolve as $D$ in order to keep the magnetic connectivity of the MC to the Sun (e.g., Démoulin \& Dasso 2009a). For the third expansion rate, $m$, we have no direct observational constraint.

Because after "S" on Ulysses (see Fig. 2) the cloud is strongly perturbed, we compare ACE and Ulysses in the inbound branch. Column 2 of Table 3 shows the mean values inside the inbound branch for the proton density $\left(n_{\mathrm{p}}\right)$ and the magnetic field observed on ACE. Column 3 shows the same mean values, but now observed on Ulysses. Columns 4-6 show three predictions for these quantities on Ulysses. The field strength, $B$, is computed from $B_{y, \mathrm{MV}}$ and $B_{z, \mathrm{MV}}$, since $B_{x, \mathrm{MV}}$ depends on the impact parameter, which differs on both spacecraft. Anyway, $\left|B_{x, \mathrm{MV}}\right| \ll B$ so that including $B_{x, \mathrm{MV}}$ does not change significantly the results. All the predictions are made using $n=1$. Column 4 (UlyP1) shows the prediction on Ulysses using $l=$ $\left(\zeta_{\text {ACE }}+\zeta_{\text {Uly }}\right) / 2=0.7$ and $m=l$ (i.e., an isotropic expansion in the plane perpendicular to the cloud axis). Column 5 (UlyP2) shows the prediction on Ulysses using $l=0.7$ and $m=1$, which corresponds to an expansion such that the cross-section of the magnetic cloud is deformed toward an oblate shape in the plane perpendicular to the cloud axis, with the major axis perpendicular to the global flow speed (e.g., Démoulin \& Dasso 2009b). Column 6 (UlyP3) shows the prediction on Ulysses using $l=0.74$ and $m=1$, which corresponds to an expansion in the direction of the plasma main flow as observed on ACE, emulating the case in which the expansion in this direction was similar to the one observed on ACE almost all the time.

The assumption $l=0.70$ and $m=l$ (UlyP1) predicts significantly higher values for all the quantities with respect to the observed ones (Table 3 ). However, the assumption $l=0.70$ and $m=1$ (UlyP2) provides more realistic predictions. Furthermore, the assumption $m=1$, combined with $l=0.74$, gives predictions closer to the observations. Of course, due to the lack of a perfect alignment, we do not expect an exact matching even when the expansion is well modeled.

Another possible approach is to compute $l, m, n$ from the observed ratio (Ulysses with respect to ACE observations) of the mean values of $n_{\mathrm{p}}, B_{y, \mathrm{MV}}$, and $B_{z, \mathrm{MV}}$ in the inbound. We find $l=0.78, m=1.04$, and $n=1.06$. The value of $l$ is close to $\zeta_{\mathrm{ACE}}$, measured independently of in situ velocity, and $n$ is close to the expected value obtained with a flux-rope length proportional to the distance to the Sun. 
Table 4. MHD quantities calculated according to each model (see Sect. 3.2).

\begin{tabular}{llccc}
\hline \hline Model & Parameter & ACE & Ulysses & \% of decay \\
\hline DM-in & $F_{z} / \mathrm{Mx} \times 10^{21}$ & 1.2 & 0.8 & 33 \\
EFI & $F_{z} / \mathrm{Mx} \times 10^{21}$ & 1.0 & 0.9 & 11 \\
\hline DM-in & $F_{y} / \mathrm{Mx} \times 10^{21}$ & 2.7 & 2.5 & 7 \\
EFI & $F_{y} / \mathrm{Mx} \times 10^{21}$ & 2.2 & 1.9 & 14 \\
\hline DM-in & $H / \mathrm{Mx}^{2} \times 10^{42}$ & -6.5 & -3.9 & 40 \\
EFI & $H / \mathrm{Mx}^{2} \times 10^{42}$ & -2. & -1.8 & 10 \\
\hline DM-in & $E / \mathrm{erg} \times 10^{28}$ & 18 & 4. & 78 \\
EFI & $E / \mathrm{erg} \times 10^{28}$ & 14.5 & 3.8 & 74 \\
\hline
\end{tabular}

Notes. The first column indicates the model, the second shows the name of the global MHD quantities and their units, the next two columns show ACE and Ulysses results, and the last column shows the percentage of decay between ACE and Ulysses results.

We conclude that the expansion of this cloud between ACE and Ulysses was such that $l \approx \zeta_{\mathrm{ACE}}$ and $m \approx n \approx 1$. From this anisotropic expansion, and assuming a circular cross section for the cloud on ACE, we predict an oblate shape on Ulysses with an aspect ratio on the order of $(5.4)^{1-\zeta_{\mathrm{ACE}}} \approx 1.55$. If this anisotropic expansion is also present before the cloud reaches $1 \mathrm{AU}$, this aspect ratio could be even a bit higher.

\subsection{Magnetic fluxes and helicity}

After fitting all the free parameters of the expanding model to the observations (method EFI), we computed the global magnetic quantities (Sect. 3.3, Eqs. (13)-(17)). We also computed these quantities from the direct observations in the inbound branch, using the direct method (see Sect. 3.4, Eqs. (18)-(21)). We use a length $L=2$ for ACE and $L=10.8$ for Ulysses, because the $\mathrm{MC}$ is still connected to the Sun when observed at $1 \mathrm{AU}$ and at 5.4 AU. There is good agreement between the magnetic fluxes and helicities found on ACE and on Ulysses, with a trend towards finding slightly lower values on Ulysses, i.e., a small decay of $\approx 7-40 \%$, depending on the method of estimating them (Table 4). We recall that the EFI method uses the full MC observations, so it is less accurate because of the strongly perturbed outbound branch.

The magnetic energy is not an MHD invariant. In fact, its decay is predicted as $e(t)^{-1}$ (Eq. (17)) assuming a self-similar expansion with $l=m=n$. For the MC studied here, we expect a decay with a factor $5.4^{-l}$. This factor is in the range $[0.19,0.31]$ for $l$ in the range $[0.7,1$.$] . In fact, the results of$ Sect. 4.6 indicate that the expansion is anisotropic $(l \approx 0.7$, $m \approx n \approx 1$.). Computing the energy decay with such anisotropic evolution would require a theoretical development that is beyond the scope of this paper. Nevertheless, the energy decay is expected to be within the above range. Since the anisotropy in the coefficients $l, m, n$ is relatively small, an approximation of the magnetic energy decay is obtained using a mean expansion of $(l+m+n) / 3=0.9$, which implies an energy decay $\approx 0.22$. From the last two rows of Table 4 , the observed decay between $1 \mathrm{AU}$ and $5.4 \mathrm{AU}$ is 0.22 and 0.26 for DM and EFI, respectively. There is excellent agreement with the theoretically expected decay, even when we have simplified the analysis to $l=m=n$ and cylindrical symmetry.

\section{Solar source of the MC}

\subsection{Searching for the solar source}

The first step in determining the MC source on the Sun is to delimit the time at which the solar event could have happened. We compute the approximate transit time from Sun to Earth using the MC average velocity on ACE (see Sect. 4.5). Considering that the cloud has traveled $1 \mathrm{AU}$ at a constant velocity of $V_{\mathrm{c}} \approx 350 \mathrm{~km} \mathrm{~s}^{-1}$ (where we neglect the acceleration, which is only important in the first stages of the CME ejection), we find $\tau \approx 1 \mathrm{AU} / V_{\mathrm{c}} \approx 5$ days. As the structure was observed by ACE starting on March 4, we searched for solar ejective events that occurred five days before, around February $28 \pm 1$ day.

From February 28 to March 1, 1998, five numbered active regions (ARs) were present on the solar disk (see top panel of Fig. 5). Only very low X-ray class flares occurred in this period of time. Most of them were class B (three on February 27, two on February 28, six on March 1), and three reached class $\mathrm{C}$ on March 1 (see the X-ray light curve from the Geostationary Operational Environmental Satellites in http: // wwW . solarmonitor. org and the corresponding list of events). Two of the last C-class events occurred in AR 8169, which was located at $\approx \mathrm{S} 21 \mathrm{~W} 74$ at the time of the flares. We found no AR associated to the observed B class flares.

We have also looked for the CMEs that occurred from February 27 to March 1 in the catalog of the Large Angle and Spectroscopic Coronagraph (LASCO, Brueckner et al. 1995) on board the Solar and Heliospheric Observatory (SOHO, see http://cdaw.gsfc.nasa.gov/CME/list). Most CMEs on those days had angular widths not over $70^{\circ}$ and originated in the eastern solar limb, except for a halo and a partial halo CME.

The halo CME first appeared in LASCO C2 on February 27 at 20:07 UT. This was a poor event inserted in the LASCO catalog after a revision in January 2006. The CME is clearly visible only in LASCO C2 running difference images (see bottom panel of Fig. 5), in particular after $\approx 22: 00 \mathrm{UT}$, when its front has already left the C2 field of view (LASCO C2 field of view from 20:07 UT until 22:08 UT is only partial). The speed of the CME leading edge seen in LASCO/C2 and C3 is $\approx 420 \mathrm{~km} \mathrm{~s}^{-1}$ from a linear fit, while a fit with a second-order polynomia provides $\approx 340 \mathrm{~km} \mathrm{~s}^{-1}$ at $20 R_{\odot}$. These values agree with the velocity measured at the front of the MC on ACE $\left(\approx 385 \mathrm{~km} \mathrm{~s}^{-1}\right)$, considering that the $\mathrm{MC}$ velocity is expected to be slightly modified by the interaction with the surrounding wind during its travel to 1 AU. Moreover, a velocity of $\approx 340 \mathrm{~km} \mathrm{~s}^{-1}$ gives a travel time of $\approx 4.5$ days, as expected.

On the other hand, the partial halo CME first appears in LASCO C2 on February 28 at 12:48 UT. Its central position angle is $236^{\circ}$ and its speed from a second-order fitting is $\approx 225 \mathrm{~km} \mathrm{~s}^{-1}$, which is too low considering the MC arrival time on ACE. Furthermore, from an analysis of images of the Extreme Ultraviolet Imaging Telescope (EIT, Delaboudiniere et al. 1995) onboard SOHO in $195 \AA$ the CME seems to be a backside event. Therefore, the halo CME is the candidate for the MC solar counterpart.

To find the source of the halo CME on the Sun, we analyzed EIT images obtained on February 27 starting $2 \mathrm{~h}$ before the halo CME appearance in LASCO C2. EIT was working in CME watch mode at that time, and therefore only images in $195 \AA$ with half spatial resolution and with a temporal cadence of $\approx 15 \mathrm{~min}$, or larger, are available. A sequence of four images with full spatial resolution in all EIT spectral bands was taken at $\approx 07: 00 \mathrm{UT}$, 13:00 UT, 19:00 UT. Furthermore, there is an extended data 

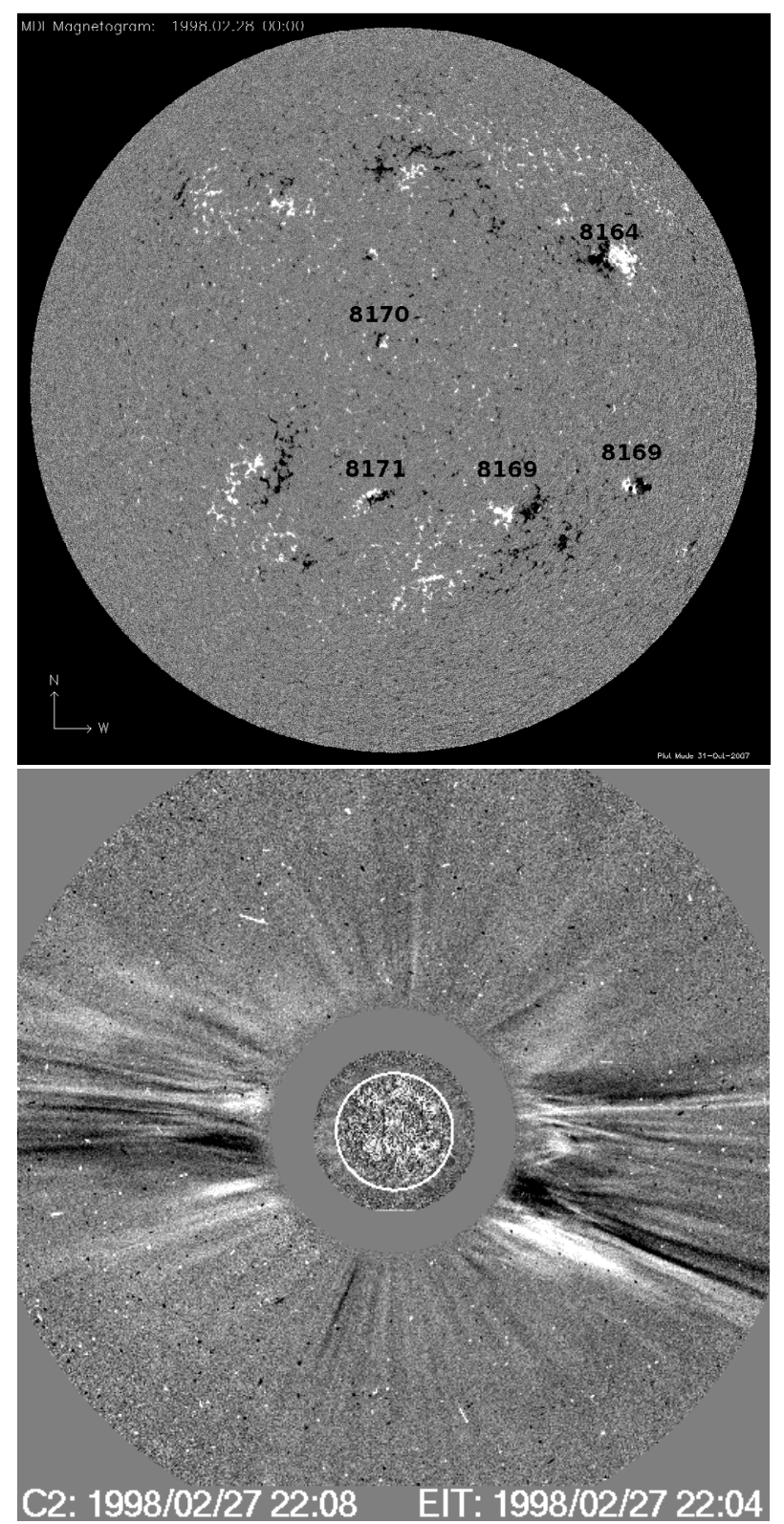

Fig. 5. (Top) MDI full disk magnetogram on February 28, 1998, at 00:00 UT (positive/negative magnetic field polarities are indicated in white/black). The five ARs present on the solar disk are indicated with their NOAA numbers. The presence of magnetic tongues with a shape compatible with a positive magnetic helicity sign is evident in AR 8171. (Bottom) LASCO C2 running difference image showing the halo CME at 22:08 UT, together with the closest in time EIT running difference image in $195 \AA$.

gap in EIT starting at around 20:00 UT until around 22:00 UT. Considering that all events on February 27 were of very low intensity (a B2.3 flare at $\approx 18: 20 \mathrm{UT}$, a B1.5 flare at $\approx 19: 17 \mathrm{UT}$, and a $\mathrm{B} 4.3$ at $\approx 22: 55 \mathrm{UT}$ after the halo CME), the low temporal cadence and spatial resolution of EIT images, and the existence of the data gap, we were not able to unambiguously identify the CME source region. However, from these images and those from the Soft X-ray Telescope (SXT, Tsuneta et al. 1991), it is evident that two of the five ARs present on the solar disk displayed signatures of activity (compare, in particular, the image at 20:17 UT with the previous and following one in SXT AlMg movie in http: //cdaw.gsfc.nasa.gov/CME_/list/); these are AR 8171 and AR 8164 (see Fig. 5).
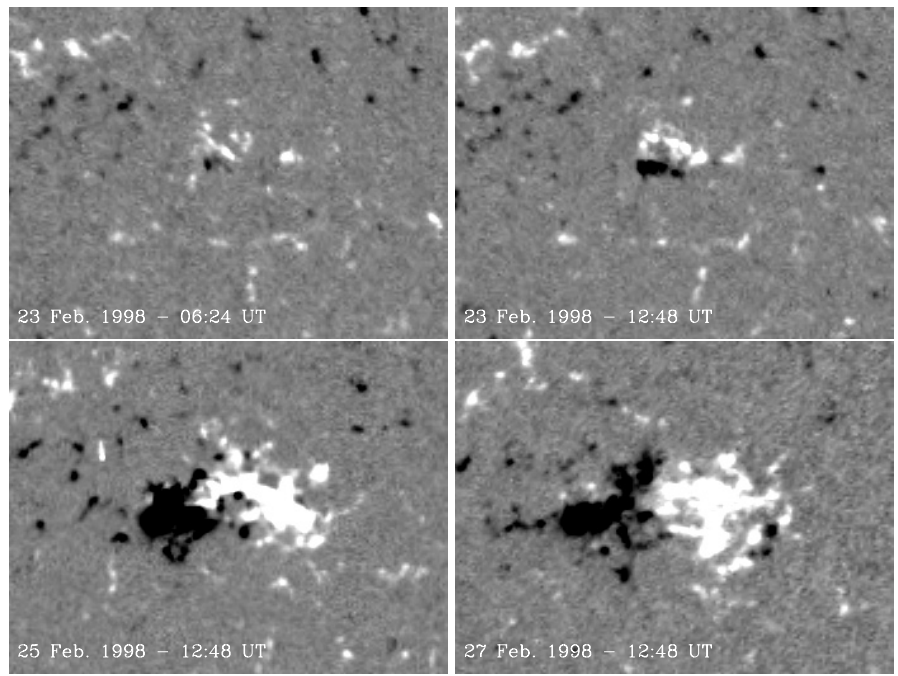

Fig. 6. MDI magnetograms of AR 8164 showing the photospheric evolution of its main polarities. The shape and evolution of the magnetic tongues indicates that the magnetic helicity of AR 8164 is negative. The magnetograms have been rotated to the central meridian position of the AR (positive/negative magnetic field polarities are indicated in white/black). The size of the field of view is the same in all panels.

From the two ARs that could be the source of the halo CME on February 27 at 20:07 UT, AR 8171 was located at S24E02, which is an appropriate location for a solar region to be the source of a cloud observed on Earth. However, the magnetic flux in this $\mathrm{AR}$ is $\approx 2.0 \times 10^{21} \mathrm{Mx}$. This value is low when compared to the range for the axial MC flux measured on $\mathrm{ACE}$ $\left(1 .-1.2 \times 10^{21} \mathrm{Mx}\right)$ since, in general, an MC axial flux is $10 \%$ of the AR magnetic flux (Lepping et al. 1997). Furthermore, according to the distribution of the photospheric field of the AR polarities (i.e., the shape of magnetic tongues, see López Fuentes et al. 2000; Luoni et al. 2011, and Fig. 5), the magnetic field helicity in this AR is positive, which is opposite the MC magnetic helicity sign. Finally, the leading polarity of AR 8171 is negative, and with a positive helicity this implies that the magnetic field component along the polarity inversion line (PIL) points from solar east to west. Since the magnetic field component along the PIL is related to the axial MC field component, this is not compatible with the MC axial field orientation on ACE that points to the solar east (Fig. 1). Therefore, we conclude that AR 8171 cannot be the solar source region of the halo CME despite its appropriate location on the disk.

The other possible CME source region is AR 8164, located at N16W32 on February 27 at $\approx 20: 00$ UT. This region is far from the central meridian. Considering this location and a radial ejection, one would expect that ACE would have crossed the ejected flux rope eastern leg; however, ACE crossed the MC front (see Sect. 4.2) which implies that during the ejection the flux rope suffered a deflection towards the east. This probably occurred low in the corona because the CME is a halo. The AR magnetic flux value is $\approx 10 . \times 10^{21} \mathrm{Mx}$, which is high enough to explain the MC axial magnetic flux as we discussed previously.

The magnetic helicity sign of AR 8164 is negative, as shown by the shape and evolution of its photospheric polarities in Fig. 6 (the spatial organization of the magnetic tongues on February 23-25). This sign is also confirmed by the coronal field model of the region (see Sect. 5.2), and it agrees with the MC helicity sign. 
Unlike AR 8171, the leading polarity in AR 8164 is positive, implying a magnetic field component pointing from west to east along the PIL. In this case, this direction is compatible with the orientation of the MC axial field. Furthermore, the PIL forms an angle of $\approx 60^{\circ}$ in the clockwise direction with the solar equator, while the MC axis lies almost on the ecliptic (see Sect. 4.2). This difference between the PIL on the Sun and the MC axial direction can be explained by a counter-clockwise rotation of the ejected flux rope, as expected, since its helicity is negative (Török \& Kliem 2005; Green et al. 2007).

From the previous analysis, we conclude that AR 8164 is the most plausible source of the halo CME on February 27, 1998, which can be the counterpart of the MC observed on ACE on March 4, 5. In the next section we compute the magnetic helicity of the AR before and after the ejection and its variation. This value is used as a proxy of the magnetic helicity carried away from the Sun by the CME.

\subsection{Physical properties of the solar source}

Using AR 8164 MDI magnetograms, we extrapolated the observed photospheric line of sight component of the field to the corona under the linear (or constant $\alpha$ ) force-free field assumption: $\boldsymbol{\nabla} \times \boldsymbol{B}=\alpha \boldsymbol{B}$. We used a fast Fourier transform method as proposed by Alissandrakis (1981) and the transformation of coordinates discussed in Démoulin et al. (1997). The value of $\alpha$ was chosen so as to best fit the observed coronal loops at a given time. We needed high spatial resolution images to identify independent loops, which are not available at times close enough before the ejection. Then, we have used the full spatial resolution images obtained by EIT in $171 \AA$ on February 27 at 13:00 UT; this is the closest time to the event in which coronal loops are visible. The boundary conditions for the model are given by the MDI magnetogram at 12:48 UT on the same day. The value of $\alpha$ is determined through an iterative process explained in Green et al. (2002). The value of $\alpha$ that best fits the observed loops is $\alpha=-9.4 \times 10^{-2} \mathrm{Mm}^{-1}$ (Fig. 7).

Once the coronal model was determined, we computed the relative coronal magnetic helicity, $H_{\text {cor }}$, following Berger (1985). In particular, we used a linearized version of the expression given by Berger (1985, see his Eq. (A23)) as in previous works by Mandrini et al. (2005) and Luoni et al. (2005). Following this approach, the magnetic helicity content in the coronal field before the ejection is $H_{\text {cor }}=-11.4 \times 10^{42} \mathrm{Mx}^{2}$.

When a flux rope is ejected from the Sun into the IP medium, it carries part of the magnetic helicity contained in the coronal field. Therefore, we have to compute the variation in the coronal magnetic helicity by subtracting its value before and after an eruptive event, to compare this quantity to the corresponding one in the associated IP event. As done before, we searched for an EIT image in $171 \AA$ with full spatial resolution after the ejection in which loops could be visible. However, in this case, the $\mathrm{AR}$ is much closer to the east limb and projection effects, added to the low intensity of the coronal structures, made it even more difficult to distinguish the shape of individual loops. As a result, $\alpha$ cannot be unambiguously determined; i.e., we can adjust the global shape of EIT brightness with more than one $\alpha$ value. We selected the EIT image at 01:20 UT on February 28 and, following a conservative approach, we determined a lower bound for the coronal magnetic helicity variation. We selected the closest in time MDI magnetogram (at 01:36 UT on February 28) and, using the previously determined value for $\alpha$, we compute $H_{\text {cor. }}$. As the AR magnetic field is decaying, its flux is lower than
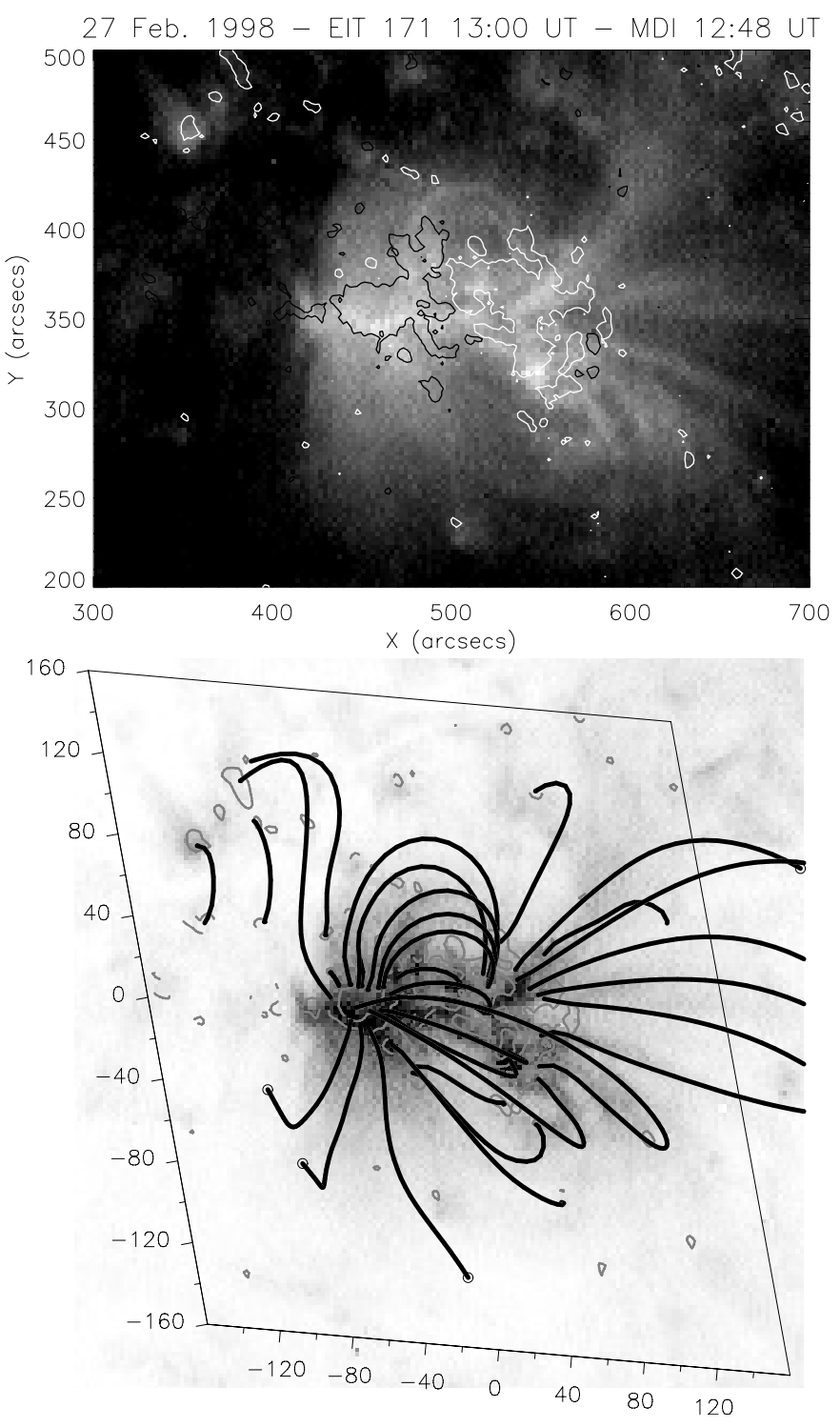

Fig. 7. (Top) EIT image in $171 \AA$ at 13:00 UT on February 27, 1998, with two MDI isocontours overlaid $( \pm 100 \mathrm{G})$ shown with continuous lines (positive: white line, negative: black line). The MDI magnetogram was taken at 12:48 UT. (Bottom) The same EIT image with more isocontours $( \pm 100,500 \mathrm{G})$ shown with gray lines and computed field lines superimposed (black continuous lines). Both axes are measured in Mm in the local solar frame.

before the $\mathrm{CME}\left(\approx 7.0 \times 10^{21} \mathrm{Mx}\right)$; therefore, $H_{\text {cor }}$ is also lower, $H_{\text {cor }}=-8.1 \times 10^{42} \mathrm{Mx}^{2}$. The real value of the coronal magnetic helicity after the CME should be even lower than the later one, as we expect that the field relaxes to a state closer to potential. To determine the range of variation for $H_{\text {cor }}$, we also computed its value taking the lowest $\alpha$ value $\left(\alpha=-6.3 \times 10^{-2} \mathrm{Mm}^{-1}\right)$ that still gives a good fitting to the global shape of EIT brightness after the CME; in this case, $H_{\text {cor }}=-5.4 \times 10^{42} \mathrm{Mx}^{2}$. Considering the two values determined for $H_{\text {cor }}$ after the CME, we estimate that $3.3 \times 10^{42} \mathrm{Mx}^{2} \leq\left|\Delta H_{\text {cor }}\right| \leq 6.0 \times 10^{42} \mathrm{Mx}^{2}$.

\subsection{Link with the observed $M C$}

From estimations of the helicity content when the cloud was observed on ACE and Ulysses, using the EFI and DM methods (see Table 4), we found $2 \times 10^{42} \mathrm{Mx}^{2} \leq\left|H_{\mathrm{MC}}\right| \leq 6 \times 10^{42} \mathrm{Mx}^{2}$, 

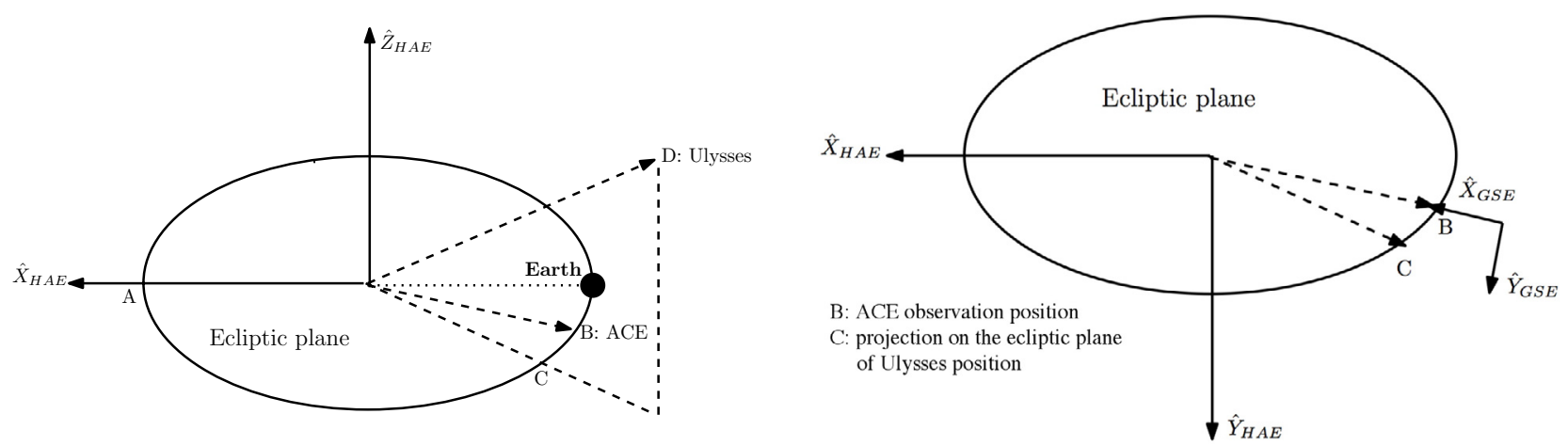

Fig. 8. Schema of the positions of each spacecraft at the observed times (left). Schema showing the relationship between the GSE and the HAE system of coordinates (right).

which is fully consistent with the range found for the release of magnetic helicity in the corona during the CME eruption.

A fraction of the total magnetic flux of $\operatorname{AR} 8164(\approx 10 \times$ $\left.10^{21} \mathrm{Mx}\right)$ is enough to account for the magnetic flux in the MC on $\mathrm{ACE}, F_{z}\left(\approx 10^{21} \mathrm{Mx}\right)+F_{y}\left(\approx 2 \times 10^{21} \mathrm{Mx}\right) \approx 3 \times 10^{21} \mathrm{Mx}$. Thus, we have found qualitative and quantitative proofs that let us associate the halo CME observed by LASCO C2 on February 27, 1998, to its solar source region (AR 8164) and to its interplanetary counterpart, the MC observed on ACE on March 4, 5, 1998, and on Ulysses on March 24-28, 1998.

\section{Summary and conclusions}

We have studied a magnetic cloud that was observed in situ by two spacecraft (ACE and Ulysses) in an almost radial alignment with the Sun $\left(\approx 2^{\circ}\right.$ for latitude and $\approx 6^{\circ}$ for longitude $)$ and significantly separated in distance (ACE at $1 \mathrm{AU}$ and Ulysses at 5.4 AU). This is an uncommon geometrical scenario, and it is very appropriate for multi-spacecraft analysis of MC evolution. In each of the spacecraft locations, we analyzed the cloud in the local frame (attached to the flux-rope axis) and quantified magnetic fluxes, helicity, and energy, using an expansion model of an initial Lundquist field (EFI) and a direct method (DM), which permits the computation of global magnetic quantities directly from the observed magnetic field (Dasso et al. 2005a). We also computed the local nondimensional expansion rate $(\zeta)$ on ACE and on Ulysses from the observed bulk velocity profiles (as defined in Démoulin et al. 2008).

We found close values of the normalized expansion rate along the solar radial direction, $\zeta_{\mathrm{ACE}}=0.74$ and $\zeta_{\mathrm{Uly}}=0.67$, as measured from the radial proton velocity on ACE and Ulysses, respectively. From the measured $\zeta_{\mathrm{ACE}}$ in the radial direction and assuming a self-similar expansion proportional to the solar distance in the orthoradial directions, we successfully predicted on Ulysses the values of the MC size, the mean values for density, and magnetic field components from the values of these quantities measured on ACE.

Next, comparing observations from Ulysses with different models for anisotropic expansions in the two directions that cannot be directly observed ( $m$ and $n$ ), we found that the expansion on the plane perpendicular to the cloud axis is larger than in the direction perpendicular to the radial direction from the Sun. Based on the quantification of this anisotropic expansion, we conclude that the initial isotropic structure at 1 AU will develop an oblate shape such that its aspect ratio would be $\approx 1.6$ at $5.4 \mathrm{AU}$ (i.e., the major axis $\approx 60 \%$ larger than the minor one, with the major one perpendicular to the radial direction from the Sun).
From a comparison of the transit time, axis orientation, magnetic fluxes, and magnetic helicity, and considering all the solar sources inside a time window, we also identified the possible source at the Sun for this event, finding an agreement between the amounts of magnetic fluxes and helicity, consistent with a rough conservation of these so-called ideal-MHD invariants.

In particular, we found that there is a small decay of the magnetic fluxes and helicity between 1 and $5.4 \mathrm{AU}$, with $\mathrm{a} \approx 10 \%$ of decay for $F_{z}$ and $F_{y}$ and a decay of $\approx 10 \%$ for the magnetic helicity when the EFI method is used and $\approx 40 \%$ when DM is used. These decays can come from a possible erosion or pealing of the flux rope during its travel, for instance, because of magnetic reconnection with the surrounding SW (e.g., Dasso et al. 2006).

For a self-similar expansion and known expansion rates, it is possible to theoretically derive the decay of the magnetic energy during the travel of the flux rope in the SW. From the observed values of $\zeta$ and modeling the expansion rates in the other two directions ( $m$ and $n$ ), we predict its decay during the travel from $1 \mathrm{AU}$ to $5.4 \mathrm{AU}$. The measurements confirm this expected magnetic energy decay (from $(15-18) \times 10^{28}$ erg to $4 \times 10^{28} \mathrm{erg}$ ).

Summarizing, in this work we validate for the first time that the local expansion rate $(\zeta)$ observed from the velocity profile can be used to predict of the decay of mass density and magnetic quantities. From the comparison of detailed predictions and observations of the decays of these quantities, we provide empirical evidence about the quantification of the anisotropic expansion of magnetic clouds beyond Earth, to 5 AUs. Finally, we quantify how much the so-called ideal-MHD invariants are conserved in flux ropes traveling in the solar wind. Then, this kind of combined study, using multi-spacecraft techniques, is a powerful approach to improving our knowledge of the properties and evolution of magnetized plasma structures ejected from the Sun.

\section{Appendix A: Transformation of coordinates between ACE and Ulysses}

We describe below the transformation of coordinates between the natural system where the vector data of Ulysses are provided $(\hat{\boldsymbol{R}}, \hat{\boldsymbol{T}}, \hat{\boldsymbol{N}})$ to the GSE system at the time when the cloud was observed by ACE. Then, we provide a common frame to compare vector observations made on Ulysses and ACE (as shown in Figs. 3, 4).

A third coordinate system, the HAE system (Heliocentric Aries Ecliptic, Fränz \& Harper 2002), is used because the location of both spacecraft are known in the HAE system. In this frame, $\hat{\boldsymbol{Z}}_{\mathrm{HAE}}$ is normal to the ecliptic plane, and $\hat{\boldsymbol{X}}_{\mathrm{HAE}}$ is positive towards the first point of Aries (from Earth to Sun at the vernal 
equinox, $\approx$ March 21). At the moment when the cloud was observed by ACE, it was located at a longitude $\epsilon_{\mathrm{A}}=163^{\circ}$ and a latitude $\tau_{\mathrm{A}}=0^{\circ}$ in the HAE system. When the MC was observed by Ulysses, this spacecraft was located at a longitude of $\epsilon_{\mathrm{U}}=157^{\circ}$ and at a latitude of $\tau_{\mathrm{U}}=2^{\circ}$.

The solar equator is defined as the plane normal to the Sun's rotation vector $(\boldsymbol{\Omega})$, and it is inclined by $\alpha \approx 7.25^{\circ}$ from $\hat{\boldsymbol{Z}}_{\mathrm{HAE}}$. In 2000, the solar equator plane intersected the ecliptic plane at an HAE longitude of $\approx 75.6^{\circ}$. Then, the angle between the projection of $\boldsymbol{\Omega}$ on the ecliptic is $\beta \approx 14.4^{\circ}$. When we write $\hat{\boldsymbol{R}}$ and $\boldsymbol{\Omega}$ in the HAE system of coordinates, we obtain

$$
\begin{aligned}
\hat{\boldsymbol{R}}= & \cos \left(\tau_{\mathrm{U}}\right) \cos \left(\epsilon_{\mathrm{U}}\right) \hat{\boldsymbol{X}}_{\mathrm{HAE}}+\cos \left(\tau_{\mathrm{U}}\right) \sin \left(\epsilon_{\mathrm{U}}\right) \hat{\boldsymbol{Y}}_{\mathrm{HAE}} \\
& +\sin \left(\tau_{\mathrm{U}}\right) \hat{\boldsymbol{Z}}_{\mathrm{HAE}}, \\
\boldsymbol{\Omega}= & \sin (\alpha) \cos (\beta) \hat{\boldsymbol{X}}_{\mathrm{HAE}}-\sin (\alpha) \sin (\beta) \hat{\boldsymbol{Y}}_{\mathrm{HAE}} \\
& +\cos (\alpha) \hat{\boldsymbol{Z}}_{\mathrm{HAE}} .
\end{aligned}
$$

To obtain $\hat{\boldsymbol{T}}$ and $\hat{\boldsymbol{N}}$ on Ulysses in HAE coordinates, we compute

$$
\hat{\boldsymbol{T}}=\frac{\boldsymbol{\Omega} \times \hat{\boldsymbol{R}}}{|\boldsymbol{\Omega} \times \hat{\boldsymbol{R}}|}
$$

$\hat{N}=\hat{R} \times \hat{T}$.

To go to the local GSE system of reference we do a last rotation around $\hat{Z}_{\mathrm{HAE}}$, which coincides with $\hat{Z}_{\mathrm{GSE}}$, in an angle $\delta=180^{\circ}-\epsilon_{\mathrm{A}}$ (see Fig. 8).

Acknowledgements. The authors thank the referee for helpful comments that improved the clarity of the paper. This research made use of NASA's Space Physics Data Facility (SPDF). We thank the ACE and Ulysses instrument teams. We thank their respective principal investigators, namely N. F. Ness (ACE/MAG), D. J. McComas (ACE/SWEPAM and Ulysses/SWOOPS), and A. Balogh (Ulysses/VHM). The authors acknowledge financial support from ECOS-Sud through their cooperative science program (No. A08U01). This work was partially supported by the Argentinean grants: UBACyT 20020090100264, UBACyT X127, PIP-2009-00825, PIP-2009-00166 (CONICET), PICT-200700856, and PICT-2007-1790 (ANPCyT). M.S.N. acknowledges Fundação de Amparo a Pesquisa do Estado de São Paulo (FAPESP - Brazil) for partial financial support. S.D. and C.H.M. are members of the Carrera del Investigador Científico (CIC), CONICET. S. Dasso acknowledges support from the Abdus Salam International Centre for Theoretical Physics (ICTP), as provided in the frame of his regular associateship.

\section{References}

Alissandrakis, C. E. 1981, A\&A, 100, 197

Attrill, G., Nakwacki, M. S., Harra, L. K., et al. 2006, Sol. Phys., 238, 117

Balogh, A., Beek, T. J., Forsyth, R. J., et al. 1992, A\&AS, 92, 221

Bame, S. J., McComas, D. J., Barraclough, B. L., et al. 1992, A\&AS, 92, 237

Berdichevsky, D. B., Szabo, A., Lepping, R. P., Viñas, A. F., \& Mariani, F. 2000, J. Geophys. Res., 105, 27289

Berdichevsky, D. B., Lepping, R. P., \& Farrugia, C. J. 2003, Phys. Rev. E, 67, 036405

Berger, M. A. 1985, ApJS, 59, 433

Bothmer, V., \& Schwenn, R. 1994, Space Sci. Rev., 70, 215

Bothmer, V., \& Schwenn, R. 1998, Annales Geophysicae, 16, 1

Brueckner, G. E., Howard, R. A., Koomen, M. J., et al. 1995, Sol. Phys., 162, 357

Burlaga, L. F. 1988, J. Geophys. Res., 93, 7217

Burlaga, L. F. 1995, Interplanetary magnetohydrodynamics (New York: Oxford University Press)

Burlaga, L. F., \& Klein, L. 1980, NASA STI/Recon Technical Report N, 80, 22221

Burlaga, L., Sittler, E., Mariani, F., \& Schwenn, R. 1981, J. Geophys. Res., 86, 6673

Cane, H. V., Richardson, I. G., \& Wibberenz, G. 1997, J. Geophys. Res., 102, 7075

Chen, J. 1996, J. Geophys. Res., 101, 27499

Cid, C., Hidalgo, M. A., Nieves-Chinchilla, T., Sequeiros, J., \& Viñas, A. F. 2002, Sol. Phys., 207, 187
Dasso, S. 2009, in IAU Symp. 257, ed. N. Gopalswamy, \& D. F. Webb, 379

Dasso, S., Mandrini, C. H., Démoulin, P., \& Farrugia, C. J. 2003, J. Geophys. Res., 108, 1362

Dasso, S., Gulisano, A. M., Mandrini, C. H., \& Démoulin, P. 2005a, Adv. Space Res., 35, 2172

Dasso, S., Mandrini, C. H., Démoulin, P., et al. 2005b, in Proc. Solar Wind 11 - SOHO 16 Connecting Sun and Heliosphere, ed. B. Fleck, \& T. Zurbuchen (European Space Agency), 605

Dasso, S., Mandrini, C. H., Démoulin, P., \& Luoni, M. L. 2006, A\&A, 455, 349 Dasso, S., Nakwacki, M. S., Démoulin, P., \& Mandrini, C. H. 2007, Sol. Phys., 244,115

Delaboudiniere, J.-P., Artzner, G. E., Brunaud, J., et al. 1995, Sol. Phys., 162, 291

Démoulin, P. 2009, Sol. Phys., 257, 169

Démoulin, P., \& Dasso, S. 2009a, A\&A, 498, 551

Démoulin, P., \& Dasso, S. 2009b, A\&A, 507, 969

Démoulin, P., Bagala, L. G., Mandrini, C. H., Henoux, J. C., \& Rovira, M. G. 1997, A\&A, 325, 305

Démoulin, P., Nakwacki, M. S., Dasso, S., \& Mandrini, C. H. 2008, Sol. Phys., 250,347

Du, D., Wang, C., \& Hu, Q. 2007, J. Geophys. Res., 112, A09101

Elliott, H. A., McComas, D. J., Schwadron, N. A., et al. 2005, J. Geophys. Res., 110, A04103

Farrugia, C. J., Burlaga, L. F., Osherovich, V. A., et al. 1993, J. Geophys. Res., 98, 7621

Farrugia, C. J., Osherovich, V. A., \& Burlaga, L. F. 1997, Annales Geophysicae, 15,152

Farrugia, C. J., Janoo, L. A., Torbert, R. B., et al. 1999, in Solar Wind Nine, ed. S. R. Habbal, R. Esser, J. V. Hollweg, \& P. A. Isenberg, AIP Conf. Proc., 471, 745

Foullon, C., Owen, C. J., Dasso, S., et al. 2007, Sol. Phys., 244, 139

Fränz, M., \& Harper, D. 2002, Planet. Space Sci., 50, 217

Goldstein, H. 1983, in Solar Wind Five, NASA CP-2280, ed. M. Neugebauer, 731

Gosling, J. T., McComas, D. J., Phillips, J. L., et al. 1995, Geophys. Res. Lett., 22,1753

Green, L. M., López fuentes, M. C., Mandrini, C. H., et al. 2002, Sol. Phys., 208, 43

Green, L. M., Kliem, B., Török, T., van Driel-Gesztelyi, L., \& Attrill, G. D. R. 2007, Sol. Phys., 246, 365

Gulisano, A. M., Dasso, S., Mandrini, C. H., \& Démoulin, P. 2005, JASTP, 67, 1761

Gulisano, A. M., Dasso, S., Mandrini, C. H., \& Démoulin, P. 2007, Adv. Space Res., 40, 1881

Gulisano, A. M., Démoulin, P., Dasso, S., Ruiz, M. E., \& Marsch, E. 2010, A\&A, 509, A39

Hammond, C. M., Crawford, G. K., Gosling, J. T., et al. 1995, Geophys. Res. Lett., 22, 1169

Hidalgo, M. A. 2003, J. Geophys. Res., 108, 1320

Hidalgo, M. A., Cid, C., Vinas, A. F., \& Sequeiros, J. 2002, J. Geophys. Res., 107, 1002

Hu, Q., \& Sonnerup, B. U. Ö. 2001, Geophys. Res. Lett., 28, 467

Kilpua, E. K. J., Liewer, P. C., Farrugia, C., et al. 2009, Sol. Phys., 254, 325

Koval, A., \& Szabo, A. 2010, J. Geophys. Res., 115, A12105

Leitner, M., Farrugia, C. J., Möstl, C., et al. 2007, J. Geophys. Res., 112, A06113

Lepping, R. P., Burlaga, L. F., \& Jones, J. A. 1990, J. Geophys. Res., 95, 11957

Lepping, R. P., Szabo, A., DeForest, C. E., \& Thompson, B. J. 1997, in Correlated Phenomena at the Sun, in the Heliosphere and in Geospace, ed. A. Wilson, ESA SP-415, 163

Lepping, R. P., Berdichevsky, D. B., Szabo, A., Arqueros, C., \& Lazarus, A. J. 2003, Sol. Phys., 212, 425

Lepping, R. P., Wu, C., Berdichevsky, D. B., \& Ferguson, T. 2008, Annales Geophysicae, 26, 1919

Liu, Y., Richardson, J. D., \& Belcher, J. W. 2005, Planet. Space Sci., 53, 3

Liu, Y., Richardson, J. D., Belcher, J. W., et al. 2006, J. Geophys. Res., 111, A12S03

Liu, Y., Luhmann, J. G., Huttunen, K. E. J., et al. 2008, ApJ, 677, L133

Lopez, R. E., \& Freeman, J. W. 1986, J. Geophys. Res., 91, 1701

López Fuentes, M. C., Démoulin, P., Mandrini, C. H., \& van Driel-Gesztelyi, L. 2000, ApJ, 544, 540

Lundquist, S. 1950, Ark. Fys., 2, 361

Luoni, M. L., Mandrini, C. H., Dasso, S., van Driel-Gesztelyi, L., \& Démoulin, P. 2005, JASTP, 67, 1734

Luoni, M. L., Démoulin, P., Mandrini, C. H., \& van Driel-Gesztelyi, L. 2011, Sol. Phys., in press

Lynch, B. J., Zurbuchen, T. H., Fisk, L. A., \& Antiochos, S. K. 2003, J. Geophys. Res., 108, 1239

Mandrini, C. H., Pohjolainen, S., Dasso, S., et al. 2005, A\&A, 434, 725 
McComas, D. J., Bame, S. J., Barker, P. L., et al. 1998, Geophys. Res. Lett., 25, 4289

Morales, L. F., Dasso, S., \& Gómez, D. O. 2005, J. Geophys. Res., 110, A04204

Möstl, C., Farrugia, C. J., Biernat, H. K., et al. 2009, Sol. Phys., 256, 427

Mulligan, T., \& Russell, C. T. 2001, J. Geophys. Res., 106, 10581

Mulligan, T., Russell, C. T., Anderson, B. J., et al. 1999a, J. Geophys. Res., 104, 28217

Mulligan, T., Russell, C. T., Anderson, B. J., et al. 1999b, in Solar Wind Nine, ed. S. R. Habbal, R. Esser, J. V. Hollweg, \& P. A. Isenberg, AIP Conf. Proc., 471,689

Mulligan, T., Russell, C. T., Anderson, B. J., \& Acuna, M. H. 2001, Geophys. Res. Lett., 28, 4417

Nakwacki, M., Dasso, S., Démoulin, P., \& Mandrini, C. 2008a, Geofisica Internacional, 47, 295

Nakwacki, M., Dasso, S., Mandrini, C., \& Démoulin, P. 2008b, J. Atm. Sol.-Terr. Phys., 70, 1318

Osherovich, V. A., Farrugia, C. J., \& Burlaga, L. F. 1993a, Adv. Space Res., 13, 57

Osherovich, V. A., Farrugia, C. J., \& Burlaga, L. F. 1993b, J. Geophys. Res., 98, 13225

Qiu, J., Hu, Q., Howard, T. A., \& Yurchyshyn, V. B. 2007, ApJ, 659, 758

Reisenfeld, D. B., Gosling, J. T., Forsyth, R. J., Riley, P., \& St. Cyr, O. C. 2003, Geophys. Res. Lett., 30, 8031
Riley, P., Linker, J. A., Mikić, Z., et al. 2003, J. Geophys. Res., 108, 1272

Riley, P., Gosling, J. T., \& Crooker, N. U. 2004, ApJ, 608, 1100

Rodriguez, L., Zhukov, A. N., Dasso, S., et al. 2008, Annales Geophysicae, 26, 213

Shimazu, H., \& Marubashi, K. 2000, J. Geophys. Res., 105, 2365

Shimazu, H., \& Vandas, M. 2002, Earth, Planets, and Space, 54, 783

Skoug, R. M., Feldman, W. C., Gosling, J. T., et al. 2000, J. Geophys. Res., 105, 27269

Smith, C. W., L'Heureux, J., Ness, N. F., et al. 1998, Space Sci. Rev., 86, 613

Sonnerup, B. U., \& Cahill, L. J. 1967, J. Geophys. Res., 72, 171

Török, T., \& Kliem, B. 2005, ApJ, 630, L97

Tsuneta, S., Acton, L., Bruner, M., et al. 1991, Sol. Phys., 136, 37

Vandas, M., \& Romashets, E. P. 2002, in Solar Variability: From Core to Outer Frontiers, ed. A. Wilson, ESA SP-506, 217

Vinas, A. F., \& Scudder, J. D. 1986, J. Geophys. Res., 91, 39

Wang, C., Du, D., \& Richardson, J. D. 2005a, J. Geophys. Res., 110, A10107

Wang, Y., Ye, P., Zhou, G., et al. 2005b, Sol. Phys., 226, 337

Webb, D. F., Lepping, R. P., Burlaga, L. F., et al. 2000, J. Geophys. Res., 105, 27251

Xiong, M., Zheng, H., Wu, S. T., Wang, Y., \& Wang, S. 2007, J. Geophys. Res., 112, A11103

Xiong, M., Zheng, H., \& Wang, S. 2009, J. Geophys. Res., 114, A11101 